\title{
Self-Motion Perception During Locomotor Recalibration: More Than Meets the Eye
}

\author{
Frank H. Durgin \\ Swarthmore College
}

\author{
Adar Pelah \\ University of York and Harvard Medical School
}

\author{
Laura F. Fox, Jed Lewis, Rachel Kane, and Katherine A. Walley \\ Swarthmore College
}

\begin{abstract}
Do locomotor aftereffects depend specifically on visual feedback? In 7 experiments, 116 college students were tested, with closed eyes, at stationary running or at walking to a previewed target after adaptation, with closed eyes, to treadmill locomotion. Subjects showed faster inadvertent drift during stationary running and increased distance (overshoot) when walking to a target. Overshoot seemed to saturate (i.e., reach a ceiling) at $17 \%$ after as little as $1 \mathrm{~min}$ of adaptation. Sidestepping at test reduced overshoot, suggesting motor specificity. But inadvertent drift effects were decreased if the eyes were open and the treadmill was drawn through the environment during adaptation, indicating that these effects involve self-motion perception. Differences in expression of inadvertent drift and of overshoot after adaptation to treadmill locomotion may have been due to different sets of ancillary cues available for the 2 tasks. Self-motion perception is multimodal.
\end{abstract}

In this article, we will consider the recalibration of locomotor actions in response to novel perceptuomotor contingencies. Although visual feedback is a powerful basis for locomotor recalibration (e.g., Rieser, Pick, Ashmead, \& Garing, 1995), self-motion perception does not cease when the eyes are closed. We show that although two forms of locomotor aftereffect can be moderated by the presence of visual feedback during adaptation, they can also be generated even when visual feedback is absent altogether. We argue that this is not because vision is irrelevant to the production

Frank H. Durgin, Laura F. Fox, Jed Lewis, Rachel Kane, and Katherine A. Walley, Department of Psychology, Swarthmore College; Adar Pelah, Department of Electronics, University of York, Heslington, York, United Kingdom, and Schepens Eye Research Institute, Harvard Medical School.

Laura F. Fox is now at the Department of Brain and Cognitive Sciences, Massachusetts Institute of Technology. Jed Lewis is now at Harvard Business School.

Experiment 1 was presented at the annual meeting of the Association for Research in Vision and Ophthalmology, Fort Lauderdale, Florida, May 2000. Experiments 1 and 2 were conducted as part of Jed Lewis's senior thesis project. Experiments 2 and 3 were presented at the 43rd Annual Meeting of the Psychonomic Society, Kansas City, Missouri, November 2002. Experiment 7 was presented at the First Annual Meeting of the Vision Sciences Society, Sarasota, Florida, May 2001.

This research was supported by a Swarthmore College faculty research grant to Frank H. Durgin, by a Howard Hughes Medical Institute summer fellowship to Laura F. Fox, and a Joel Dean summer fellowship to Jed Lewis. We thank Jack Loomis for helpful comments on a draft of this article. Aimee Schultz developed the treadmill controller used in Experiments 4-6. Donald Reynolds constructed the moving treadmill apparatus used in Experiment 7. Lee Paczulla and Evan Schaffer assisted with the collection of data for Experiments 4-6.

Correspondence concerning this article should be addressed to Frank H. Durgin, Department of Psychology, Swarthmore College, 500 College Avenue, Swarthmore, PA 19081. E-mail: fdurgin1@swarthmore.edu of these aftereffects but because the perception of self-motion is multimodal: Even when the eyes are closed during adaptation, perceptual information is available that can be used to estimate self-motion and to recalibrate locomotor action accordingly.

Following a practice developed by earlier researchers, we used fairly high locomotor speeds for the purpose of maximizing cue conflict between locomotor signals (signifying rapid forward selfmotion) and other perceptual signals that specify an absence of self-motion during treadmill locomotion. Thus, it should be understood that we were seeking to modify the calibration state of a subset of the available self-motion signals by altering the relationship between these various sources of information during adaptation. We postulated that recalibration may occur in response to discrepancies between individual estimators (e.g., visual, vestibular, haptic, kinesthetic, and motor estimators) and the resulting perception of self-motion. If one source of information is in disagreement with the others, that source may be recalibrated the most, but it may also drive the other signals somewhat. It remains possible that there are direct intersignal recalibrations that occur as well. We return to a discussion of this aspect of our theory below.

Self-motion perception has been historically regarded as involving vestibular proprioceptors. But as Gibson (1966) pointed out, there are a variety of sensory inputs to the perception of selfmotion, including optic flow. Lishman and Lee (1973) showed that when optic flow specifying linear self-motion was put in competition with vestibular inputs, it tended to dominate them, even in cases of extreme discrepancy (see also Sun, Campos, \& Chan, 2004). There remain those who advocate for strong vestibular inputs to linear self-motion perception-especially in the absence of vision (Harris, Jenkin, \& Zikovitz, 2000; Israël, Grasso, Georges-François, Tsuzuku, \& Berthoz, 1997; Marlinsky, 1999), but the vestibular system, because it operates on inertial inputs, turns out to be a noisy estimator of velocity (Schaffer \& Durgin, 2005). Studies of self-motion perception in which passive motions 
differ from walking speed have shown biases to perceive passively experienced speed as nearer to walking speed than it is (Mittelstaedt \& Mittelstaedt, 2001). This can be interpreted as indicating the existence of a strong Bayesian prior (i.e., walking speed) in the estimation of self-motion, but it may also simply reflect a (possibly cognitive) strategy of estimating distance by time-rather than perceiving distance directly by vestibular integration. In any case, it emphasizes the relative poverty of the inertial detectors as means of estimating the velocity (or distance) of whole-body self-motion. Indeed, stimulation of the vestibular otoliths during forward acceleration is ambiguous between forward acceleration and backward tilt. This ambiguity has been shown to be resolved, in part, by cognitive factors (Wertheim, Mesland, \& Bles, 2001). It may contribute to the inherent weakness of the vestibular estimator.

Although vision dominates in the control of locomotion for most humans, studies of walking without visual feedback demonstrate remarkably precise calibration in walking to distant targets (Loomis, Da Silva, Fujita, \& Fukusima, 1992; Thomson, 1983). That is, having visually previewed a target as much as $20 \mathrm{~m}$ away (Rieser, Ashmead, Talor, \& Youngquist, 1990), a person can close his or her eyes and walk to it fairly accurately and without bias. Indeed, walking without visual feedback is one of a number of indirect measures of distance perception (including throwing without feedback) at which humans perform especially well (e.g., Thomson, 1983; see Loomis \& Beall, 2004, for a review). This task is regularly used to assess distance perception itself, but what is it that allows for such precise control of self-motion?

To answer this question, it is useful to divide potential selfmotion information into two classes. The first class refers to internally derived signals that are relevant to self-motion. These are often called idiothetic (e.g., Mittelstaedt \& Mittelstaedt, 2001) or interoceptive, and they are provided by internally generated sensations, including efference copy (von Holst \& Mittelstaedt, 1950), vestibular signals, and kinesthetic proprioception (e.g., Riley \& Turvey, 2001). The second class of self-motion information, called allothetic or exteroceptive, is provided by vision, audition, and haptic contact with environmental features, among other things. An account that places exclusive emphasis on the visual and motor components of self-motion perception neglects to consider the many remaining kinds of interoceptive and exteroceptive perceptual information. All of these sources of information may be integrated in an internal model (Wolpert, Ghahramani, \& Jordan, 1995) of whole-body motion with respect to the physical environment. We suspect that recalibration of locomotor activity takes place with respect to this internal model, though it is also possible that recalibration takes place pairwise between various sources of information.

For the purpose of exposition, we initially assume (and do not attempt to prove) a Bayesian cue-combination model in our reasoning about the integration and recalibration of sensory information concerning self-motion. In such a model, various estimators are combined using weights that reflect the reliability of those estimators. Estimators that consistently disagree with the overall perception of self-motion (show bias) are recalibrated to reduce the discrepancy. Visual cues to spatial position are probably extremely difficult to override (leading to visual dominance) when they are sufficiently immersive. Similarly, the evidence of Mittelstaedt and Mittelstaedt (2001) can be interpreted as demonstrating that noninertial interoceptive sources of information (e.g., kinesthetic sen- sation and efference copy) tend to dominate vestibular inputs with regard to perceived distance of self-motion in the absence of vision. Included in our model, however, are such haptic cues to nonmotion as contact with the rails of the treadmill, which (perhaps in combination with cognitive awareness and the absence of inertial cues) seem to strongly dominate locomotor cues when a person walks on a treadmill.

\section{Two Aftereffects of Self-Motion Perception}

Here, we compare two locomotor aftereffects that seem to involve recalibration of self-motion perception (probably by altering the motor-kinesthetic estimates). Anstis (1995) showed that after running on a treadmill, subjects who attempted to run in place with their eyes closed would drift forward inadvertently. We refer to this as the drift effect. We consider that it represents an error in self-motion perception. The other locomotor aftereffect that we discuss was demonstrated by Rieser et al. (1995), and we refer to it as the overshoot effect. Rieser et al. showed that when subjects walked on a treadmill at one speed and the treadmill itself was drawn through the world at a different speed, subsequent locomotor calibration was changed. Specifically, when later trying to walk to a visually previewed target, subjects would overshoot the target if they had previously adapted to walking on a treadmill at a speed that was faster than the speed at which they were being pulled through the world. Rieser et al. also found evidence of undershoot when the pulling speed was greater, though this effect was less pronounced and received less study. The overshoot effect is also an error of self-motion perception (as is the undershoot effect). We discuss the overshoot effect in more detail before discussing the drift effect.

Rieser et al. (1995) seemed to provide evidence that vision was necessary to produce their aftereffect by showing that no reliable overshoot occurred after simple treadmill walking with closed eyes (i.e., there was no visual input and, hence, no aftereffect). As we discuss below, however, this experiment did not serve as an adequate control for the role of vision because the treadmill speeds used were only about half those used in Reiser et al.'s other experiments. Higher treadmill speeds ought to produce stronger recalibration effects than lower speeds, because discrepancies between locomotor activity and perceived self-motion are greater. Our experiments show that large overshoot effects occur even with eyes closed during adaptation when higher treadmill speeds are used.

\section{Varieties of Functional Adaptation}

Rieser et al. (1995) further argued that locomotor action is calibrated to visual feedback in a functional way. Their principal conclusion was that the recalibration they found generalized to actions with the same functional goal. Specifically, they demonstrated that the recalibration of forward walking transferred to sidestepping (i.e., toward the goal of advancing in the same direction via locomotion) but not to throwing. They argued that adaptation was specific to the functional goal of locomotion. Because "functional" is too general a term, we refer to this as goal-specific recalibration.

It is not clear that goal-specificity is the best way to interpret Rieser et al.'s (1995) findings, however. For example, they dis- 
cussed circumstances in which an altered environment might reasonably change the calibratory state across a variety of control systems, such as in the presence of a strong wind. But some environmental conditions are more specific than others. For example, the traction available on surfaces varies, but this would probably affect locomotion more than it would (underhanded) throwing. The pattern of results that Rieser et al. presented is consistent with the idea of calibration to a specific surface. Such calibration ought to be limited to activities in which traction is relevant, but it should not transfer to throwing. Thus, the transfer to sidestepping is also consistent with altering one's representation of certain properties of the external environment. We refer to this notion as environmental recalibration. It might be thought of as involving updating internal models of the affordances of the external environment. A recent example is the broken escalator effect (Reynolds \& Bronstein, 2003), which may be a form of contingent adaptation (Durgin, 1996).

However, a third kind of "functional" basis for calibration is the common need for biological perceptual systems to stay actively calibrated in the presence of internal noise and calibration drift. In the case of walking, for example, various internal conditions (including limb growth, e.g., or neural change) could affect the mapping of motor commands to actual locomotor progress. Thus, recalibrating the internal models of perception-action systems (such as the self-motion system; see Gibson, 1966) would be yet another kind of recalibration. Such changes in calibration would be registered by perceptual systems monitoring self-motion, and they would be in response to errors in the internal model of self-motion. Note that only partial transfer to sidestepping might be expected under such a model, because the action of each limb and muscle system would need to be individually calibrated to account for its particular dynamics. We might describe this kind of recalibration as recalibration of the internal model of body dynamics with respect to self-motion, but we will refer to it simply as organismic recalibration to contrast it with environmental recalibration.

Obviously, a system that responds to mismatches between motor predictions of self-motion and consequent perceived self-motion could accomplish both environmental and organismic recalibration. But patterns of generalization would be expected to differ. Rieser et al. (1995) used the patterns of transfer to other kinds of activity to argue that the recalibration was goal specific rather than organismic. However, a one-legged hopping variant of the overshoot effect has recently been reported as a consequence of treadmill hopping, and this aftereffect is entirely limb specific (Durgin, Fox \& Kim, 2003; see also Gordon, Fletcher, Melvill Jones, \& Block, 1995). Such limb specificity is predicted by an organismic recalibration theory but is inconsistent both with the goal-specific recalibration account favored by Rieser et al. and with environmental recalibration. Moreover, as the experimental data presented below show, the results reported by Rieser et al. concerning transfer to sidestepping are actually quantitatively consistent with the idea that transfer was only partial, also supporting the organismic view of recalibration.

\section{Reinterpreting the Drift Effect}

Let us turn now to Anstis's (1995) drift effect. Anstis found that after running on a treadmill for as little as $1 \mathrm{~min}$, subjects who attempted to run in place with their eyes closed would inadver- tently drift forward. Anstis did not interpret this aftereffect in terms of self-motion perception but, rather, as a kind of motor adaptation to the backward motion of the treadmill belt. Because the effect could be generated on a treadmill with eyes open or closed and was not generated by simply running around on solid ground, Anstis argued that the effect had nothing to do with the perception of self-motion. It is important to understand that Anstis did not refer to locomotor recalibration at all in his explanation of the drift effect. Instead, he showed that there was no interlimb transfer for the hopping version of the drift effect and, consequently, concluded that the effect was an entirely motoric adaptation of gait (perhaps in conjunction with limb proprioception) that was specific to the use of a treadmill or similar device.

Durgin and Pelah (1999) have shown that Anstis (1995) was incorrect in his conclusions regarding the origins of the drift effect. They adapted subjects to running on solid ground with their eyes open or closed and showed that running with closed eyes on solid ground was sufficient to generate a strong drift effect. This suggests that the treadmill belt is not exclusively responsible for the drift effect and that self-motion perception is, in fact, relevant after all. Similarly, Durgin et al. (2003) produced leg-specific hopping drift aftereffects by adapting subjects to one-legged forward hopping on solid ground with closed eyes. These effects did not occur following hopping with open eyes.

Thus, our understanding of the overshoot effect and the drift effect is that both seem to be organismic recalibrations in response to situations in which perceptual expectancies (resulting from running, hopping, or walking activity) conflict with the perceived sense of self-motion. That is, we agree with Rieser et al. (1995) that these aftereffects are due to a kind of conflict between what we would call motor predictions (e.g., Wolpert \& Flanagan, 2001) and the perception of self-motion. However, we disagree with Rieser et al.'s assertion that these effects are goal specific rather than limb specific. Instead, we understand the effects to be due to recalibrations of motor predictions for specific motor systems (though environmentally contextualized aspects of the effects are possible).

Moreover, whereas Rieser et al. (1995; see also Pick, Rieser, Wagner, \& Garing, 1999) and Anstis (1995) both seemed to believe that their aftereffects must either involve vision exclusively or be strictly motoric, our view is that this is a false dichotomy and that both aftereffects involve a more broadly construed concept of self-motion perception. Thus, whereas Pick et al. (1999), for example, argued that perceptuomotor learning can only take place with open eyes and concluded that any adaptation with closed eyes must be "sensory" adaptation, our position is quite different. Vision is but one source (albeit an important one) of perceptual information for self-motion perception. If there are internal models of the motion of the whole body through space, then those models incorporate more than just purely motor and visual information (see Loomis \& Beall, 2004), and perceptuomotor learning can take place with eyes closed (Durgin \& Pelah, 1999).

\section{A Multimodal Model of Locomotor Recalibration}

Both the drift and overshoot aftereffects might be understood, most parsimoniously, as the result of a recalibration of the perception of self-motion from motor-kinesthetic senses. This recalibra- 
tion occurs in response to cue conflict between motor-kinesthetic senses and other sources of self-motion information (Durgin et al., 2003). Specifically, both overshoot and drift are predicted if estimates of the rate of self-motion provided by the motor system (and/or limb proprioception) are lower than the actual resulting self-motion. In the case of the drift aftereffect, the forward motor estimate is zero while the actual forward motor output (result) is slightly positive. In the overshoot aftereffect, the forward motor estimate is again slightly less than the actual forward motor result. Although we cannot demonstrate all aspects of our theory in this article, we can at least establish that the recalibrations that produce drift and overshoot are more closely related than has been previously argued. We show that both effects can be produced without vision and that both are affected by visual information. Further, we argue that both are best understood as organismic recalibrations of kinesthetic estimates of self-motion with respect to perceived self-motion.

The strong version of our theory supposes that perceived selfmotion serves as a kind of clearinghouse into which all the various estimates are funneled and combined and with respect to which all the various estimates are then recalibrated (when they disagree with the result of that combination). However, for the present purposes, it is equally valid to suppose that estimates provided by various sensory systems enter into direct comparisons as well or instead. It is certainly simpler to imagine that the recalibration is guided by appeal to the "best" (integrated) estimate at any given time (and such a notion is supported by evidence that, e.g., visual flow is not always compelling). In any case, if two cues strongly disagree, then one or both of them will disagree with the ultimate perception of self-motion and may be subject to recalibration. For this reason, we sometimes speak of conflict between cues as leading to recalibration without meaning to take a stand on whether it is direct conflict or conflict mediated by the central perception of self-motion that produces recalibration. We suspect that both routes to recalibration are present.

\section{Experimental Strategy}

The experiments reported in this article were primarily designed to compare and contrast the drift and overshoot aftereffects, and the results contribute to the growing evidence that both of these effects are organismic recalibrations of motor estimates of selfmotion. Our first two experiments show that the adaptation procedure developed by Anstis (1995) can be used to produce Rieser et al.'s (1995) overshoot effect. That is, people will overshoot targets in a blind walking task after running on a treadmill with closed eyes for as little as $1 \mathrm{~min}$. This result was previously reported by Durgin, Pelah, and Amiruddin (1998), but they did not include a control condition for which there was no adaptation. Our second experiment, in which we examine the effect of adaptation time on the overshoot effect, suggests that there may be a ceiling on the amount of overshoot that can be obtained using a treadmill. After contrasting this with the drift aftereffect in Experiment 3, which shows no evidence of a ceiling for these brief adaptation times, we then develop three experiments that bear on characterization of the effects of treadmill running. The first two of these look for evidence of transfer to sidestepping after $1 \mathrm{~min}$ (Experiment 4 ) and after 5 min (Experiment 5) of treadmill running. These two experiments support our contention that transfer to sidestep- ping is quite limited and variable and is probably interpretable in terms of organismic recalibration. These experiments also support the notion that the amount of overshoot generated during forward walking seems to rapidly saturate (i.e., reach a ceiling), because similar effect sizes are found for both 1 and 5 min of adaptation in the forward conditions of those experiments. Experiment 6 looks at the time course of the development of the overshoot aftereffect by means of repeated adaptations and shows that a reasonably large aftereffect can be generated in as little as $20 \mathrm{~s}$ of treadmill running.

In the third and seventh experiments, we seek to better characterize the drift aftereffect. Our third experiment shows that the drift effect is not subject to the same ceiling effect as the overshoot effect, though we point out that this difference in the expression of the two effects may be superficial. Finally, the seventh experiment is designed to demonstrate that even treadmill-induced versions of the drift aftereffect can be modified when enhanced visual selfmotion information is supplied. We show that the adaptation procedure developed by Rieser et al. (1995), in which the environmental speed is faster than the treadmill speed, reduces the size of Anstis's (1995) drift aftereffect, as predicted by cue-conflict theory (Durgin et al., 2003; Durgin \& Pelah, 1999). This is important because it is the first time that aftereffects of treadmill running (rather than running on solid ground) have been reduced by supplying real environmental motion.

\section{Methodological Concerns}

In addition to the theoretical points we address, there are several methodological and interpretive issues that risk complication of our exposition. We hope that by addressing them here, we can prevent confusion later. Both Rieser et al. (1995) and Anstis (1995) published data that we believe have led to misunderstandings of the nature of their respective effects. We list the two main problems here, and we also discuss an additional measurement problem.

\section{Why So Slow?}

Rieser et al. (1995) reported that visual experience was necessary to create the overshoot effect. In their control experiment, however, in which subjects adapted to walking on a normal treadmill with closed eyes (and showed no reliable overshoot effect), the treadmill speeds used were much lower than (about half) the speeds used in all of their other adaptation experiments. We believe that a very different conclusion would have been reached had Rieser et al. used the same speeds in the control experiment as they did in their main experiments. Although we used treadmill running (following Anstis, 1995) rather than walking in the present experiments, we have replicated our results with fast treadmill walking. There is plenty of overshoot after treadmill walking with eyes closed.

We note that Rieser et al. (1995; like Anstis, 1995) seemed to believe that aftereffects from treadmill walking with eyes closed are evidence of simple motor adaptation (see also Pick et al., 1999). We do not share that view, because we believe that even with closed eyes, people have an experience of whether they are moving or not. During treadmill locomotion, there is rarely any illusion that one is actually moving forward. This experience, or its sensory correlates, might be sufficient to drive perceptuomotor 
adaptation in response to the conflict between motor estimates of self-motion and the perceptual feedback received.

\section{Why Were They Decelerating?}

Anstis (1995) showed graphs of the drift effect in which the rate of drift decreased during the $15 \mathrm{~s}$ of testing. He interpreted this deceleration as evidence of a rapid decay of the aftereffect. However, he attached a spring-loaded measuring tape to the back of his subjects to measure their drift. Unless his subjects intentionally compensated for the pull of the tape, some deceleration would be an expected consequence of the backward force exerted by the measuring tape. We did not tether our subjects, and our graphs of drift show no deceleration over a $20 \mathrm{~s}$ period. This helps to characterize the drift effect as a misestimate of motor speed (rather than of acceleration or of distance).

\section{Taking Full Measure?}

A third kind of methodological issue emerges in Experiment 2, and we anticipate it here. As it turns out, the amount of overshoot demonstrated in almost all of the experiments conducted by Rieser et al. (1995), and in all of the overshoot experiments that we have conducted using treadmills, is fairly consistent in magnitude. That is, even when seemingly relevant parameters such as adaptation speed and adaptation time are varied, the amount of overshoot aftereffect seems to quickly saturate at about $17 \%$. A ceiling effect of this sort complicates the interpretation of data in some respects, but it also facilitates interpretation in others. For example, Rieser et al. found an overshoot of $9 \%$ in their experiment on sidestepping (after adaptation to $10 \mathrm{~km} / \mathrm{h}$ forward walking on a treadmill being pulled at $5 \mathrm{~km} / \mathrm{h}$ for $8 \mathrm{~min}$ ) and compared that with the (surprisingly low) $6 \%$ overshoot found for forward walking in that particular experiment. They concluded that transfer was complete. However, if the $9 \%$ value for sidestepping is compared with the $17 \%$ and $18 \%$ overshoot found for forward walking in Rieser et al.'s other experiments that used the same adaptation parameters, it appears that the transfer to sidestepping may be only partial, not complete. (It is important to note that Rieser et al., 1995, did not comment on the small effect size, though they emphasized that more of the visual world was blocked by the tractor used in this experiment than by those used in their others. However, because environmental flow was supposed to be slower than walking speed in that experiment, providing less environmental motion ought to have increased the effect.)

\section{Why the Asymmetry?}

Rieser et al. (1995) noted an apparent asymmetry in locomotor recalibration effects insofar as the overshoot that they obtained from conditions in which locomotor speed outpaced environmental speed was much greater than the undershoot obtained when environmental speed outpaced locomotor speed. Their eyes-closed control experiment was designed to show that the asymmetry was not due to simple motor adaptation (as they construed it). It was that control experiment that seemed to indicate that visual feedback was necessary during adaptation. However, had that "control" experiment used locomotor speed parameters more in line with those of their other experiments, it is likely that because of the ceiling effect we have noted, Rieser et al. would instead have found that treadmill adaptation with closed eyes could have accounted for the entire effect of overshoot, as we show below. This is not to support Rieser et al.'s alternative "motor" interpretation but to point out that much of their argument concerning the interpretation of the asymmetry observed rested on a poorly designed control experiment (i.e., one using motor parameters unlike those used in their main experiment). Although the asymmetry is not a topic of much concern in this article, we note here that one excellent suggestion (T. Shipley, personal communication, May $16,2003)$ is that the asymmetry may be a reflection of prior probabilities: There are many surfaces, such as sand or gravel, that afford less traction and require greater motor effort, but there are few surfaces (moving sidewalks?) that actually add speed or traction. On the basis of prior experience, people may therefore be more prepared to increase than to decrease their motor effort in response to the error signals produced during adaptation to conflicting perceptual and motor signals concerning the rate of self-motion.

\section{A Note on Treadmills}

Although both Anstis (1995) and Rieser et al. (1995) used treadmills for adaptation, they both seemed to overlook an important feature of treadmill locomotion. Whether the eyes are open or closed, the only way to avoid injury on a normal treadmill is for one to have a sense of where one is with respect to the treadmill. If the eyes are open, vision can accomplish this, and one can let go of the treadmill handles. With eyes closed, however, one must simply hold on to the treadmill. This means that haptic contact with the treadmill handles provides information about self-motion (or a lack thereof). Indeed, the tuning of motor activity on a treadmill is largely directed at remaining stationary with respect to the treadmill so as not to fall off.

Although a treadmill is a useful means for dissociating locomotor activity from perceived self-motion, it does not provide a neutral context for the perception of self-motion, because the primary spatial framework for the control of locomotion becomes the treadmill itself. Consequently, drawing the treadmill through the world, as Rieser et al. (1995) did, would not necessarily have overruled the fact that locomotion was being controlled with respect to the treadmill, not the passing world. This could have contributed to the asymmetry in overshoot-undershoot that Rieser et al. observed, because at least part of the representation of self-motion may have included stasis with respect to the treadmill itself. What it certainly means is that closing one's eyes while on a treadmill does not eliminate self-motion perception, because the perception of self-motion relative to the treadmill is still being used to control locomotion.

We begin with a demonstration that overshoot can be produced by adaptation to treadmill locomotion with closed eyes. This is consistent with cue-conflict theory insofar as locomotor activity on a treadmill is accompanied by the conflicting perception that one is not actually going anywhere. This conflicting perception is present whether the eyes are kept open or not. 


\section{Experiment 1: Anstis's (1995) Adaptation Procedure Produces Rieser et al.'s (1995) Aftereffect}

Rieser et al. (1995) reported that adaptation to walking on a treadmill with closed eyes produced no reliable change in performance of the task of walking to a previewed target with closed eyes. However, the treadmill speed they used to establish this null effect was much slower $(4 \mathrm{~km} / \mathrm{h})$ than the treadmill speed used in their main experiment $(8 \mathrm{~km} / \mathrm{h})$. Durgin et al. (1998) have reported preliminary evidence that a reliable overshoot aftereffect is produced by treadmill running with or without vision. Using a more advanced design, in Experiment 1 we sought to replicate that result and to produce Rieser et al.'s aftereffect (overshoot) from Anstis's (1995) adaptation procedure (1 min of treadmill running with closed eyes).

\section{Method}

Subjects. A total of 18 Swarthmore College undergraduates participated in this experiment in exchange for payment or partial fulfillment of a course requirement. Subjects were selected without regard to sex, and all had normal or corrected-to-normal vision. Assignment to experimental conditions was random.

Walking-to-target task. Unlike Rieser et al. (1995), we did not provide our subjects with the opportunity to practice walking to targets without vision, because we wanted to assess adaptation-induced changes in each subject's untrained calibration state. (It is important to note that Durgin et al., 1998, did use a training procedure. Their results were similar.)

To ensure quick and easy blindfolding at the appropriate times, subjects wore a blindfold (opaque goggles) throughout the experiment, pushing it up to their foreheads when vision was required. Subjects also wore foam earplugs (noise-reduction rating 31) to minimize usage of auditorylocalization information.

At the start of each blind walking assessment, subjects stood in a long hallway and viewed a target (a person) $8.0 \mathrm{~m}$ in front of them. They were aware that they would be required to walk to the location of this target person without the aid of vision. They then lowered the blindfold over their eyes and walked forward, stopping when they believed they had reached the place where the target person (who stepped to the side) had been standing. To keep themselves from veering off course as they walked, subjects grasped a tightly strung guidewire spanning the length of the hallway. The experimenter concurrently videotaped their feet against markings on the hallway floor so that the length and number of steps that they took could be accurately measured in addition to total distance walked toward the target.

Adaptation. Between blind walking assessments, subjects in the experimental condition ran on a Landice 8700 Sprint treadmill for $1 \mathrm{~min}$ at a speed of $9.1 \mathrm{~km} / \mathrm{h}$, blindfolded, grasping the handrails for support. Subjects in the control condition just stood on the treadmill for $1 \mathrm{~min}$, blindfolded.

Design and procedure. We used a simple pretest-adaptation-posttest design to determine the effects of treadmill adaptation on subjects' blind walking performance. The entire procedure was explained prior to the start of the experiment. Then, baseline performance on the blind walking task was assessed for all subjects. A single trial with a target at $8.0 \mathrm{~m}$ was used. Next, half of the subjects (the experimental group) completed the treadmillrunning adaptation, and the other half (the control group) simply stood on the treadmill. Blind walking performance was then reassessed with a further single trial. (We used single trials so as to avoid any contamination due to adaptation to the test itself [see Philbeck, O'Leary, \& Lew, 2004]). Subjects were subsequently debriefed and thanked.

The primary dependent measure used in this experiment was distance walked. Number of steps taken and stride length were also analyzed.

\section{Results}

Pretest walking distances $(M=7.5 \mathrm{~m})$ did not differ between the control and experimental groups, $t(16)=0.39, p>.10$, nor were these reliably different from the target distance of $8.0 \mathrm{~m}$. As anticipated, the mean posttest walking distance for the experimental group $(8.9 \mathrm{~m})$ was reliably longer than the mean pretest walking distance for that group $(7.6 \mathrm{~m}), t(8)=6.34, p<.01$. This mean adapted posttest walking distance was also longer than the mean posttest walking distance for the control group $(7.4 \mathrm{~m}), t(8)=$ $2.39, p<.05$. The mean posttest walking distance for the control group did not reliably differ from the mean pretest walking distance for that group. It is clear from these results that adaptation to treadmill running with closed eyes does produce an overshoot aftereffect on the blind walking task (see Figure 1).

Our video data also allowed us to ask whether treadmill adaptation affected stride length (arguably a motor variable) or perceived progress per step (arguably a spatiomotor variable). That is, were subjects in the experimental group taking longer strides during the posttest or simply more of them? Rieser et al. (1995) had concluded that their adaptation affected the number of steps taken by subjects but not the stride length. In our analysis as well, the increased distance walked seemed to be reflected primarily in the number of steps taken. For the experimental group, this number increased from 14.8 to $16.3, t(8)=2.40, p<.05$. No such increase was present for the control group (see Figure 2).

Although stride lengths also seemed to increase slightly with time for both groups (overall average stride length increased from 54 to $57 \mathrm{~cm}, t[17]=2.51, p<.05)$, they did so in a manner consistent with increased confidence in this unusual task. That is, the increased stride length occurred for the control group as well as the experimental group.

\section{Discussion}

It appears that Anstis's (1995) adaptation procedure is sufficient to induce the overshoot aftereffect discovered by Rieser et al. (1995). The size of the effect, expressed as a percentage of increase in distance walked, is $18 \%$. This effect size is comparable to those reported by Rieser et al. as resulting from adaptation to treadmill locomotion while being towed at a speed slower than that of the treadmill belt. Also, as Rieser et al. found, the longer walking distances after adaptation are not simply due to a purely motor variable, such as stride length, but are the result of subjects taking more steps when trying to reach the previewed target. This is more consistent with a misperception of self-motion in the distance advanced with each stride.

It would seem that Rieser et al. (1995) failed to discover an overshoot aftereffect to closed-eyes treadmill adaptation simply because they used an abnormally slow treadmill speed. That slower speed was sufficient to induce an overshoot effect in their subjects who adapted to treadmill walking with their eyes open (for $10 \mathrm{~min}$ ) but not for those who adapted with their eyes closed. In a follow-up experiment (that would be redundant to report here), we have confirmed that adapting even for just $1 \mathrm{~min}$ with closed eyes to treadmill walking at a speed of $7 \mathrm{~km} / \mathrm{h}$ is sufficient to produce a strong overshoot aftereffect.

Although a visual overestimation of the previewed distance (i.e., a change in static visual perception) could also lead to apparent 


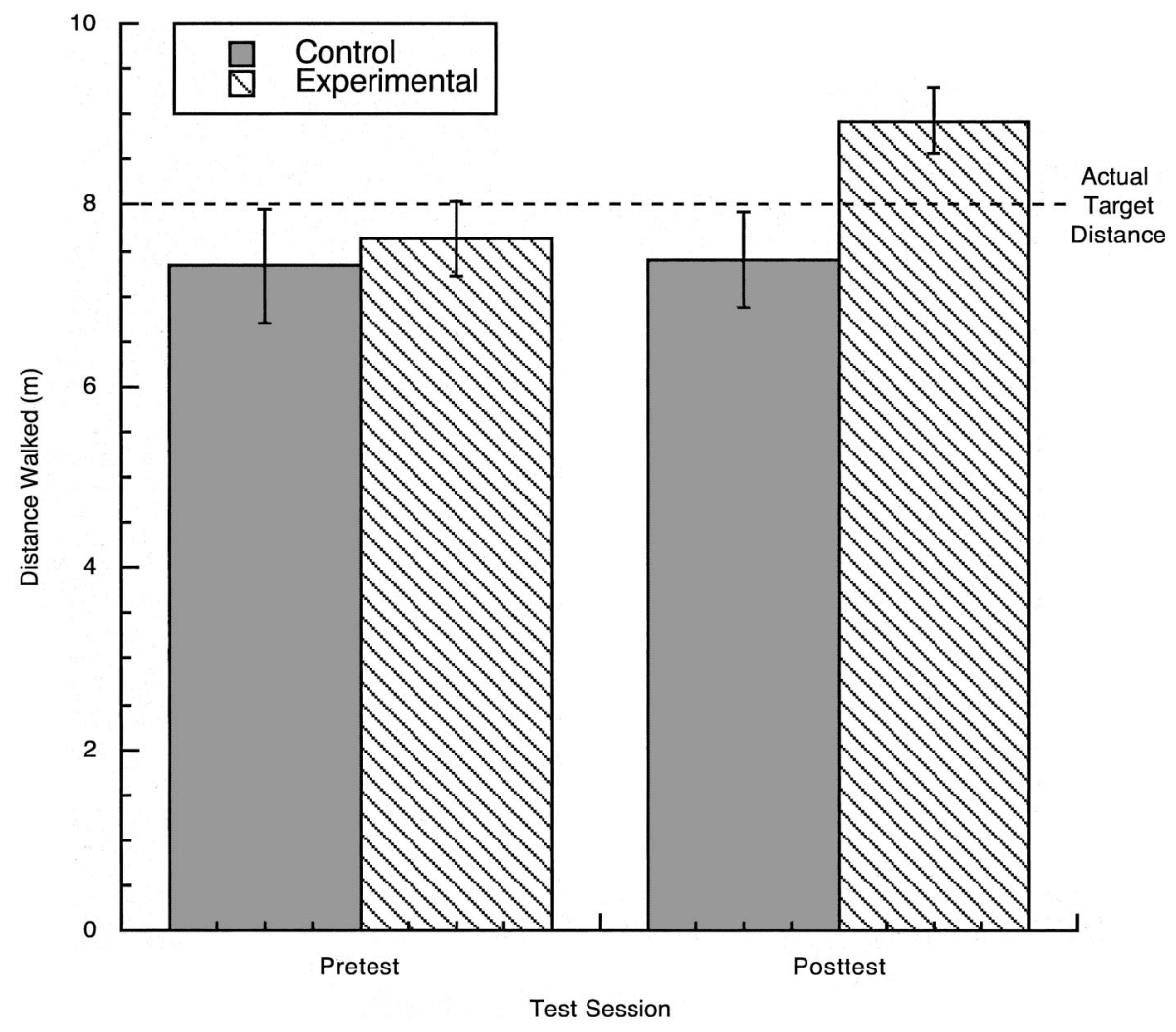

Figure 1. Mean distances walked before (pretest) and after (posttest) adaptation in the control and experimental conditions of Experiment 1. Error bars represent standard errors, plotted with respect to within-subject differences.

overshoot, and some have suggested that visual distance itself is misperceived after treadmill locomotion (Proffitt, Stefanucci, Banton, \& Epstein, 2003), there are many findings that seem to contradict this interpretation. For example, Rieser et al. (1995) found no transfer of walking overshoot to a throwing task, and Durgin et al. (2003) found no interlimb transfer for an analogous hopping task after subjects hopped on a treadmill. Because the adaptation procedure used by Proffitt et al. (2003) involved walking while looking off to one side at either a stationary virtual world (without appropriate motion parallax) or a moving virtual world (with motion parallax), the differences that they found in verbal distance estimates (of visual static scenes) between the two adaptation conditions might have been due to differential visual adaptation to depth cues in the virtual reality system, not a consequence of the concomitant motor activity.

Finally, we emphasize that the treadmill speeds we used were essentially the same as those used by Rieser et al. (1995) in all of their moving-treadmill experiments. Although Rieser et al. referred to their subjects as "walking" at speeds as high as $10 \mathrm{~km} / \mathrm{h}$ $(6 \mathrm{mi} / \mathrm{h})$, we suspect that their subjects' gaits must have been rather different from a normal walk. For example, the average speed at which they reported that their subjects walked during the testing phase of their Experiment 5 was about $1.34 \mathrm{~m} / \mathrm{s}$, which is a reasonably rapid pace $(4.8 \mathrm{~km} / \mathrm{h}$, or $3 \mathrm{mi} / \mathrm{h})$, but it is only about half the treadmill speed $(10 \mathrm{~km} / \mathrm{h})$ used in that experiment during adaptation. We used these speeds because we believed that they ought to maximize the discrepancy between motor estimates of self-motion and the concurrent perception of stasis.

\section{Experiment 2: Overshoot as a Function of Adaptation Time}

The overshoot effect produced in Experiment 1 was quite large (over a meter), and it was similar in size not only to those effects found by Rieser et al. (1995) but also to those observed by Durgin et al. (1998). Durgin et al. (1998) attempted to parametrically compare drift and overshoot aftereffects by varying visual information (eyes open or eyes closed) and treadmill speed. Although they found that the drift aftereffect was greater when adaptation occurred with open eyes (consistent with the notion that visual estimates of self-motion also contribute to adaptation) and was somewhat greater when adaptation speed was higher, they found no reliable differences in overshoot as a result of these manipulations (they found trends in the expected direction). However, the null effects that Durgin et al. (1998) found for speed parameters on overshoot might have been due to measurement variability. In the present experiment, we modified the testing procedure to eliminate sideways drift during walking (by using a guidewire, as in Experiment 1), and we tested whether $2 \mathrm{~min}$ of treadmill adaptation would produce greater overshoot than $1 \mathrm{~min}$ of treadmill adaptation. 


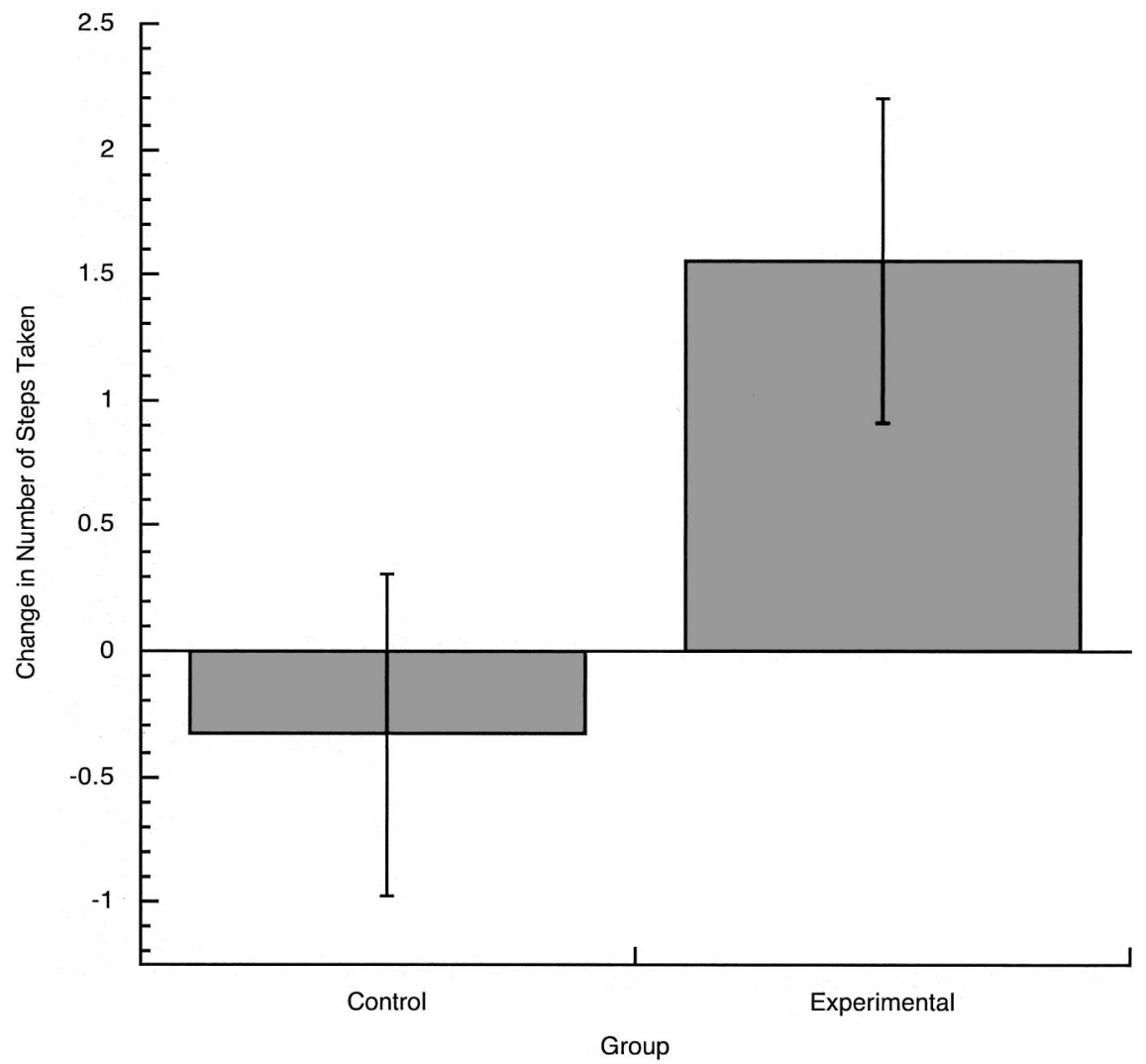

Figure 2. Mean change in number of steps taken in the control and experimental conditions of Experiment 1. Error bars represent standard errors for each group.

\section{Method}

Subjects. A total of 18 Swarthmore undergraduates participated in this experiment in exchange for payment or partial fulfillment of a course requirement. Subjects were selected without regard to sex, and all had normal or corrected-to-normal vision. Assignment to experimental conditions was random.

Walking-to-target task. As in Experiment 1, performance on a blind walking-to-target task was assessed (with single trial of $8.00 \mathrm{~m}$ ) before and after adaptation. The task was identical to that described in Experiment 1, except that instead of videotaping subjects' feet, the experimenter simply placed small stickers on the floor where subjects stopped. These stickers were later used to measure the distance that subjects had walked during the task.

Adaptation. As in Experiment 1, adaptation consisted of a period of running on the Landice treadmill at $9.1 \mathrm{~km} / \mathrm{h}$ while blindfolded, grasping the handrails for support. Ten of the subjects completed a 1-min adaptation period, and eight completed a 2-min adaptation period.

Design and procedure. The design and procedure were identical to those used in Experiment 1, except that all subjects were adapted to treadmill running for either 1 or $2 \mathrm{~min}$.

\section{Results}

A $2 \times 2$ (Test Session $\times$ Adaptation Time) repeated measures analysis of variance showed that, overall, there was an increase in walking distance from 7.87 to $9.20 \mathrm{~m}$ after adaptation, $F(1,16)=$ 13.5, $p<.01$. Moreover, the pretest walking distances (7.74 and
$8.03 \mathrm{~m}$ for the 1-min and 2-min conditions, respectively) did not differ reliably from the true target position of $8.00 \mathrm{~m}, t(17)=0.45$, $p>.1$, but posttest walking distances $(9.34$ and $9.02 \mathrm{~m}$ for the 1 -min and 2-min conditions, respectively) did, $t(17)=3.02, p<$ .01 . This replicates the basic finding of Experiment 1. However, there was no Adaptation Time $\times$ Test Session interaction $(F<1)$. That is, the amount of increase in walking distance did not reliably differ as a function of adaptation time (see Figure 3).

\section{Discussion}

As in Experiment 1, an overshoot aftereffect occurred as a result of treadmill running while blindfolded. The size of the aftereffect (17\%) was again similar to what was reported by Rieser et al. (1995). However, there was no increase in aftereffect size as a result of increasing the adaptation time from 1 to $2 \mathrm{~min}$. Although this replicated Durgin et al.'s (1998) failure to find reliable differences in overshoot on the basis of seemingly relevant variables, the trend this time was not even in the expected direction.

Table 1 shows the overshoot sizes reported by Rieser et al (1995) in relation to the various experimental parameters that they used as well as some other results, including those of this article and of the closed-eyes conditions of Durgin et al. (1998). It is evident from the table that, although there is some variability in aftereffect strengths in the Rieser et al. (1995) data, overshoot aftereffects following treadmill locomotion averaged $17 \%$ ( $S D=$ 


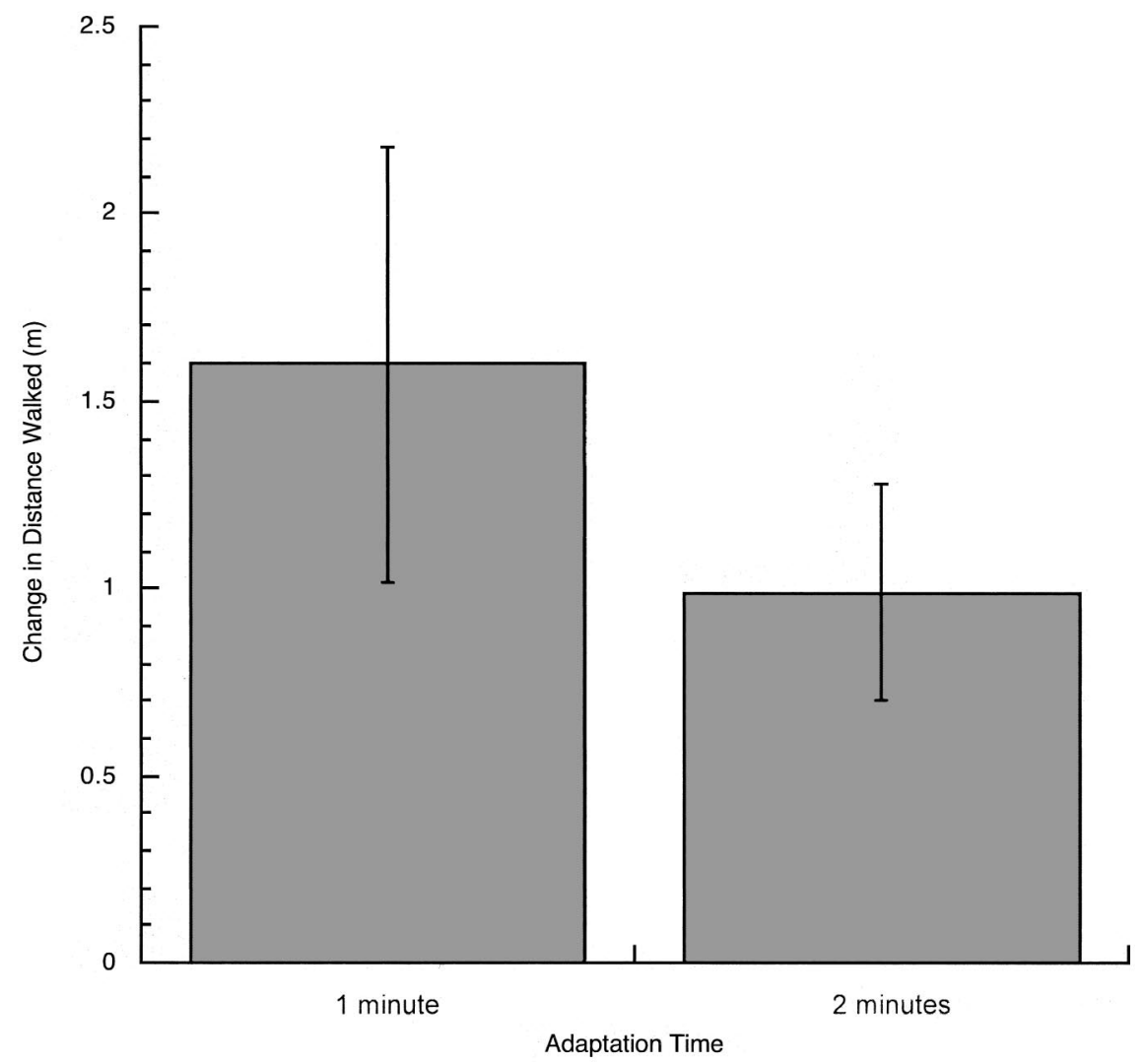

Figure 3. Mean change in distance walked as a function of adaptation time in Experiment 2. Error bars represent standard errors for each group.

3\%) whatever the adaptation time. The similarity of effect sizes with closed eyes to that with open eyes is not evidence that vision never matters; we know that when Rieser et al.'s visual speed was faster than the treadmill speed, they found undershoot. The similarity does suggest, however, that there may be a ceiling on overshoot aftereffects and that the ceiling may be reached after just 1 min of running on a treadmill. We return to this issue in later experiments.

\section{Experiment 3: Drift as a Function of Adaptation Time}

Before proceeding to interpret the apparent ceiling on the overshoot effect, we wished to test whether the strength of the drift aftereffect would be affected by adaptation time beyond $1 \mathrm{~min}$. Because Durgin et al. (1998) did detect reliable, though small, differences in the drift effect (but not the overshoot effect) as a result of small changes in treadmill speed during adaptation, we sought to determine whether changing the adaptation time would also produce differences in the drift aftereffect.

We also sought to characterize the velocity profile of drift before and after adaptation. As mentioned in the introduction, Anstis (1995) reported that the rate of forward drift decreased with time over $15 \mathrm{~s}$ of testing. According to Anstis, this deceleration was evidence of aftereffect decay. However, his subjects were attached to a spring-loaded measuring tape. It is therefore possible that a small force was drawing them backward as they moved, causing deceleration over time. In this experiment, we improved on Anstis's method by using the technique of videotaping the feet of our subjects, rather than attaching them to a measuring tape, to measure drift over time.

\section{Method}

Subjects. A total of 18 Swarthmore undergraduates participated in this experiment in exchange for payment or partial fulfillment of a course requirement. Subjects were selected without regard to sex, and all had normal or corrected-to-normal vision. Assignment to experimental conditions was random

Running-in-place task. Performance on a blind running-in-place (RIP) task was assessed before and after adaptation. A sturdy $2-\times 8-\mathrm{m}$ mat was marked with a high-contrast square grid of $8-\times 8$-cm boxes, and additional lines marked 4-cm intervals for the first $2 \mathrm{~m}$. After being blindfolded, so that they would be unaware of the presence of the grid, subjects were led a few steps through a doorway to this mat. They then attempted to run in place for $20 \mathrm{~s}$, as timed by the experimenter. During this 20 -s period, the experimenter videotaped subjects' feet against the grid. Measurements of distance drifted were later recorded from the videotape at 1-s intervals. If a subject drifted off the mat during testing (several veered to the side), data were recorded up to the departure point so that velocity could still be computed from the video record.

Adaptation. As in Experiment 2, adaptation consisted of a period of running on the Landice treadmill at $9.1 \mathrm{~km} / \mathrm{h}$ while blindfolded, grasping the handrails for support. Half of the subjects completed a 1-min adaptation period, and the other half completed a 2-min adaptation period. 
Table 1

Summary of Previous and Present Overshoot Effect Sizes as a Function of Motor Speed, Visual Speed, and Duration of Adaptation

\begin{tabular}{lcccc}
\hline & \multicolumn{2}{c}{ Speed $(\mathrm{km} / \mathrm{h})$} & & \\
\cline { 2 - 3 } \multicolumn{1}{c}{ Source } & Motor & Visual & $\begin{array}{c}\text { Adaptation } \\
(\mathrm{min})\end{array}$ & $\begin{array}{c}\text { Overshoot } \\
(\%)\end{array}$ \\
\hline Rieser et al. (1995) & & & & \\
$\quad$ Experiment 1 & 8.0 & 5 & 8.0 & 18 \\
Experiment 4 & 8.0 & 4 & $8.0^{\mathrm{a}}$ & 25 \\
Experiment 5 & 8.0 & 4 & $8.0^{\mathrm{a}}$ & 15 \\
Experiment 6 & 10.0 & 5 & $8.0^{\mathrm{a}}$ & 17 \\
Experiment 8 & 10.0 & 5 & $8.0^{\mathrm{a}}$ & 6 \\
Experiment 9 & 10.0 & 5 & $8.0^{\mathrm{a}}$ & 18 \\
Durgin et al. (1998) & 10.0 & $0^{\mathrm{b}}$ & 1.5 & 16 \\
Durgin et al. (1998) & 8.3 & $0^{\mathrm{b}}$ & 1.5 & 14 \\
Withagen \& Michaels (2002) & 6.0 & 3 & 15.0 & 13 \\
Present study & & & & \\
Experiment 1 & 9.1 & $0^{\mathrm{b}}$ & 1.0 & 18 \\
Experiment 2 & 9.1 & $0^{\mathrm{b}}$ & 1.0 & $17^{\mathrm{c}}$ \\
Experiment 2 & 9.1 & $0^{\mathrm{b}}$ & 2.0 & $17^{\mathrm{c}}$ \\
Experiment 4 & 9.1 & $0^{\mathrm{b}}$ & 1.0 & 12 \\
Experiment 5 & 9.1 & $0^{\mathrm{b}}$ & 5.0 & 16 \\
\hline
\end{tabular}

Note. Overshoot represents the change in distance walked at posttest relative to pretest.

${ }^{\text {a }}$ The actual duration was not reported by Rieser et al. (1995); the assumption here is that it was similar to that in their Experiment 1. ${ }^{\mathrm{b}}$ Eyes were closed during adaptation, so visual speed is represented as zero. ${ }^{\mathrm{c}}$ The actual 1-min change in Experiment 2 was 20\%, and the actual 2-min change was $12 \%$, but because differences between conditions were not reliable, the average change is reported for both.
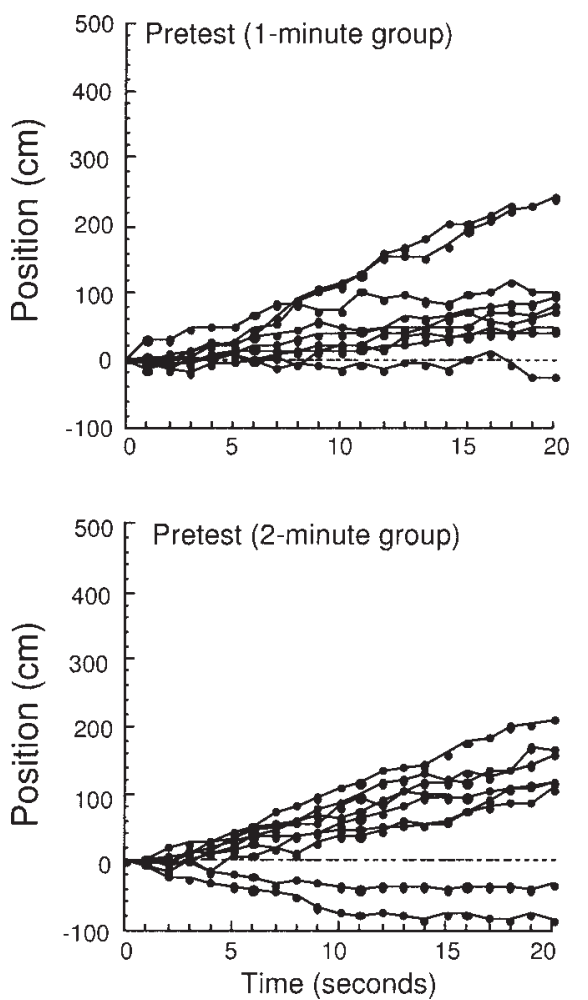

Design and procedure. A simple pretest-adaptation-posttest design was used to determine the effects of adaptation on subjects' RIP performance. Subjects wore a blindfold and foam earplugs throughout the entire experiment. Prior to the start of the experiment, the procedure was explained and subjects donned the blindfold and earplugs. Then, subjects were led to the testing mat, and baseline performance on the RIP task was assessed. Next, subjects were led to the treadmill to complete their assigned adaptation period ( 1 or $2 \mathrm{~min}$ ). On completion of adaptation, subjects were led back to the testing mat, and RIP task performance was reassessed. Subjects were subsequently debriefed and thanked.

\section{Results}

Our first question concerned the shape of the position plot over time, because this shape would determine how the data were to be analyzed. Plots of individual progress over time are shown in Figure 4. These plots, suggesting a linear change in position with time, indicate no concavity that would be evidence of gradual deceleration during the 20 -s measurement period.

Individual velocity plots were computed for each subject by taking the slope of position over time for each 3-s interval of time. When averaged (missing values were ignored), these produced the velocity profiles plotted in Figure 5. These (low-pass-filtered) profiles suggest that overall accelerations are completed within the first 3 or $4 \mathrm{~s}$. By computing the slope of position over time from $4 \mathrm{~s}$ on for each individual, we estimated drift velocity for each subject for the pretest and posttest. It is evident that there is no overall deceleration in these plots.

A repeated measures analysis of variance was conducted on the velocities, with adaptation time (1 $\mathrm{min}$ or $2 \mathrm{~min}$ ) as a between-
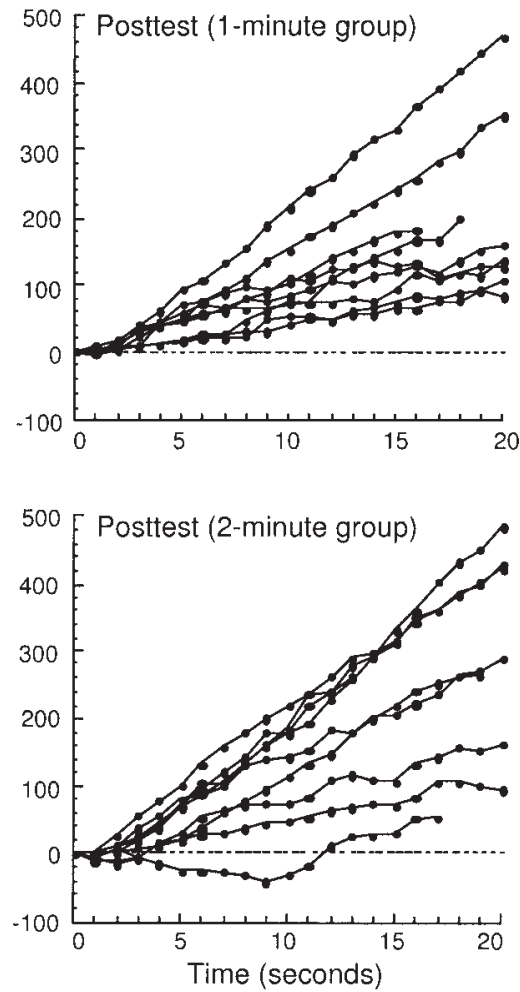

Figure 4. Inadvertent drift of each subject, shown as position over time in the pretest and after 1 or 2 min of adaptation (posttest) to treadmill running in Experiment 3. 


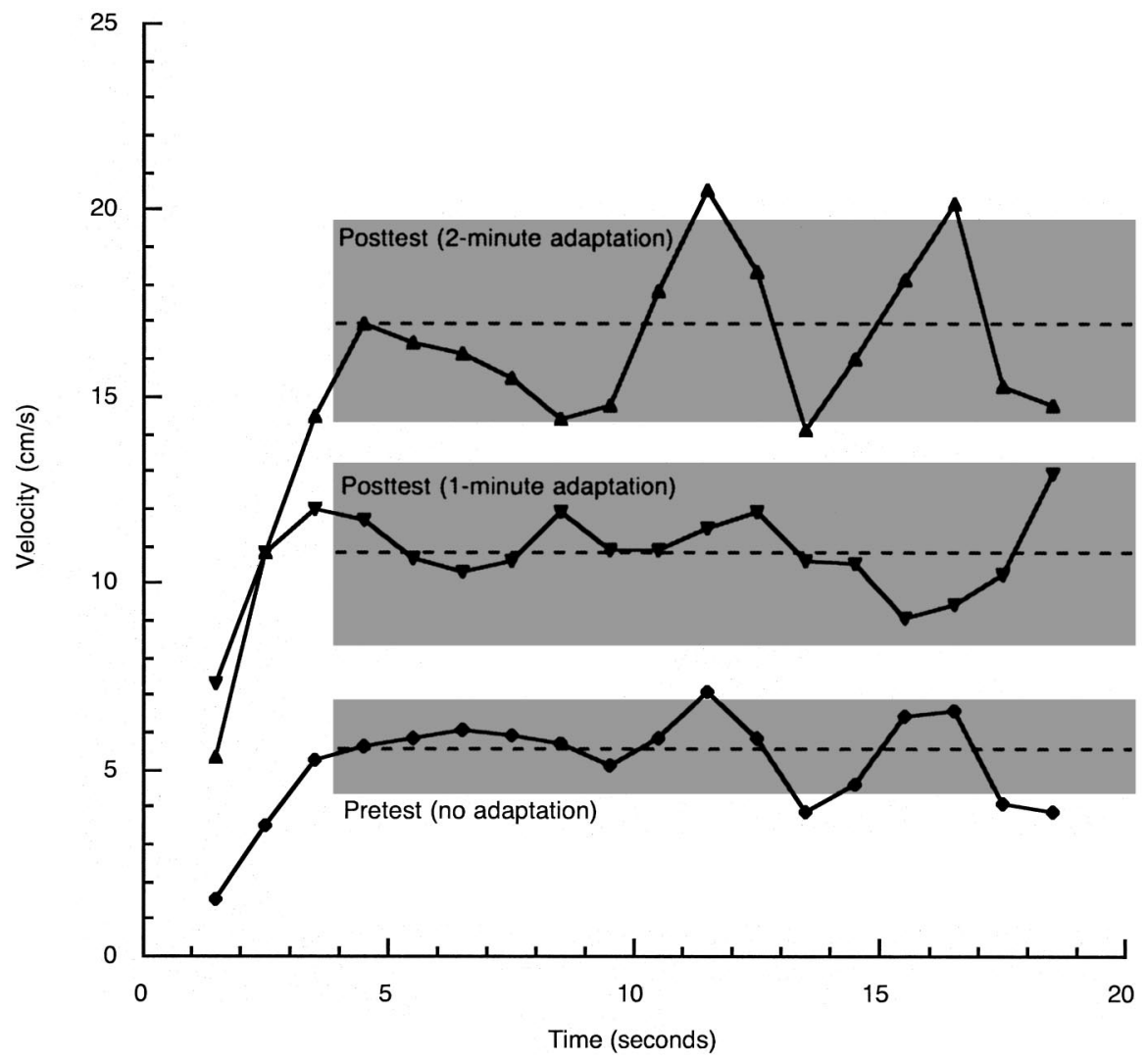

Figure 5. Averaged velocity profiles for drift in Experiment 3. Points are based on slopes fit to position plots over a 3-s window. Gray regions represent mean velocity (overall slope of each position plot) from $4 \mathrm{~s}$ on plus or minus 1 standard error.

subjects variable and test session (pretest or posttest) as a withinsubject variable. Mean pretest velocities $(5.6 \mathrm{~cm} / \mathrm{s})$ for the two groups were about equal. However, there was a reliable Adaptation Time $\times$ Test Session interaction, $F(1,16)=7.28, p<.05$. In the posttest, both the 1-min adaptation group $(10.9 \mathrm{~cm} / \mathrm{s}), t(8)=3.67$, $p<.01$, and the 2 -min adaptation group $(16.9 \mathrm{~cm} / \mathrm{s}), t(8)=6.23$, $p<.01$, showed increases relative to their pretest scores. Moreover, expressed as an increased velocity, the drift aftereffect showed by the 2 -min group $(+11.3 \mathrm{~cm} / \mathrm{s})$ was reliably greater than that shown by the 1 -min group $(+5.2 \mathrm{~cm} / \mathrm{s}), t(16)=2.70, p<.05$. Note that $11 \mathrm{~cm} / \mathrm{s}$ is equivalent to $0.40 \mathrm{~km} / \mathrm{h}$, or approximately $4 \%$ of the adapting velocity, and represents about $2 \mathrm{~m}$ of forward advance over $20 \mathrm{~s}$.

\section{Discussion}

Two clear findings emerge from this experiment. The first is that the drift aftereffect seems to involve a fairly constant velocity of drift and shows no evidence of deceleration over the $20 \mathrm{~s}$ we measured. This is consistent with the idea that locomotor aftereffects to cue conflict concerning self-motion result in a recalibration of motor-kinesthetic estimates of speed of self-motion. As discussed in the introduction, this characterization of the drift aftereffect fits well with the idea of organismic recalibration of the motor estimates of self-motion in response to cue conflict between different sources of information about self-motion.

A common interpretation of the drift and overshoot aftereffects has been made more complicated, however, because the drift aftereffect was affected by adaptation time whereas the overshoot aftereffect was not. Without knowing why there is a ceiling effect on overshoot, it is unclear whether the underlying recalibrations that produce drift and overshoot are actually the same. In theory, the motor estimate of self-motion might be similarly affected in both cases, but part of that effect may be masked, in the case of the walking task, by additional (unadapted) information about selfmotion (such as inertial signals from the vestibular otoliths) that would play a very different role in the task of running in place than it would in walking to a target.

There are at least three other plausible explanations of why the overshoot effect might reach a ceiling even though drift does not. First, walking is a much more common activity than stationary running. It may be that the recalibration of perceived speed while walking is limited because of the calibrational inertia resulting from extensive experience with walking. After all, subjects showed inadvertent drift in the stationary running task even before adaptation, whereas pretest performance in the walking task was fairly accurate even without explicit training. Just as we suggested that asymmetries in the amount of overshoot and undershoot 
obtained by Rieser et al. (1995) might have been due to differential prior probabilities for the direction of adaptation, the prior probabilities for estimating the speed of self-motion from walking may be more firmly entrenched than those for stationary running.

Second, it is possible that in walking to a previewed target, subjects somehow estimate an upper limit on how much time it might take to walk toward it on the basis of the calibration inertia of daily experience in related tasks described above. Once subjects are actually walking, time rather than distance traveled (or remaining) becomes the limiting factor when they at some point decide that surely they have walked long enough for the previewed distance. Note that this would not occur in the experimenterdelimited RIP task. If the idea that inertial (passive) estimates of self-motion velocity are probably biased toward a default speed (Mittelstaedt \& Mittelstaedt, 2001) is taken seriously, this temporal upper-bound idea can be treated as a variant of the vestibular estimator idea.

Another kind of explanation might derive from differences in the goals of the two tasks relative to the adaptation procedure. As we pointed out in the introduction, locomotor control during treadmill running is designed to keep the runner stationary with respect to the treadmill. It is therefore possible that Rieser et al. (1995) were partly correct in emphasizing goal specificity. Transfer to walking might reach a ceiling because of differences in the goals of walking to a target and running in place. Conversely, running in place has a goal similar to that of treadmill running: to jog without actually moving.

Rieser et al.'s (1995) chief argument in favor of goal specificity was transfer to sidestepping. The next two experiments that we report investigated transfer to sidestepping using our closed-eyes adaptation paradigm. These experiments have the additional virtue of contrasting brief periods of adaptation (1 min in Experiment 4) with somewhat longer periods (5 min in Experiment 5).

\section{Experiment 4: No Transfer to Sidestepping After 1 Min of Treadmill Running}

As discussed in the introduction, Rieser et al.'s (1995) strongest argument for their goal-specific recalibration came in the form of evidence that adaptation to forward walking transferred to sidestepping but not to throwing. Although they reported that they measured the same amount of overshoot with sidestepping as they did with forward walking, the actual amount found (9\%) was evidently lower than the overshoot reported in most of their other experiments. We sought to determine whether we could find evidence of equivalent transfer to sidestepping after $1 \mathrm{~min}$ of adaptation to treadmill running with eyes closed.

\section{Method}

Subjects. A total of 8 Swarthmore undergraduates participated in this experiment in exchange for payment. Subjects were selected without regard to sex, and all had normal or corrected-to-normal vision.

Design and procedure. All subjects were adapted to treadmill running twice. One time, they were tested for changes in forward walking with eyes closed; the other time, they were tested for sidestepping to a target. Order was counterbalanced.

Walking-to-target task. As in Experiment 1, performance on a blind walking-to-target task was assessed before and after adaptation. Subjects were blindfolded and wore foam earplugs, and they walked using a guidewire by grasping a plastic tube that slid along the guidewire. Subjects were tested with both forward walking and sidestepping. Because the walking task was repeated a total of four times, we varied target distance randomly on each trial between 7 and $9 \mathrm{~m}$ by increments of $10 \mathrm{~cm}$. The dependent measure was the proportion of the target distance walked. A HiBall optical position sensor was used to record starting, stopping, and target locations.

Adaptation. Adaptation consisted of a 1-min period of running on a NordicTrack Apex6100xi treadmill at $9.1 \mathrm{~km} / \mathrm{h}$ while blindfolded, grasping the handrails for support. Each subject was adapted two times to forward running, with a 1-min break between sessions. Treadmill speed was controlled by a computer interface that allowed rapid acceleration to the desired speed.

\section{Results}

Mean proportions of target distance walked are plotted in Figure 6 as a function of session (pre- or postadaptation) and manner of walking (forward or sidestepping). In this experiment, subjects systematically undershot the target distances $(M=0.79)$ prior to adaptation, $t(7)=6.06, p<.01$. There was no difference between sidestepping and forward walking in the pretest, $t(7)=0.27, p>$ .10. Following adaptation to forward running, the proportion of target distance sidestepped did not increase, $t(7)=0.24, p>.10$ (one-tailed), whereas that for forward walking did, $t(7)=2.99$, $p<.05$ (two-tailed). Changes in distances walked were greater for forward walking than for sidestepping, $t(7)=2.07, p<.05$ (one-tailed).

\section{Discussion}

After only 1 min of adaptation to treadmill running with eyes closed, there was no evidence of transfer of adaptation to sidestepping. This result is consistent with organismic recalibration, because the act of sidestepping involves a different muscular motion than does forward walking. This result is not consistent with environmental recalibration or with goal-specific adaptation. Just as Durgin et al. (2003) found no interlimb transfer of the recalibration of hopping to targets, we found no evidence of transfer to sidestepping from adaptation to forward treadmill locomotion. However, given the results of Rieser et al. (1995), it is somewhat surprising that absolutely no adaptation was found. For this reason, and to contribute to our data concerning ceiling effects, we decided to conduct a second experiment, using longer adaptation times.

\section{Experiment 5: Transfer to Sidestepping After 5 Min of Treadmill Running}

Because Rieser et al. (1995) adapted their subjects for at least 5 min, rather than 1 , we sought to determine whether a longer adaptation time would produce more evidence of transfer to sidestepping. Because of the longer period of required treadmill running, only one adaptation period was used for each subject. Changes in sidestepping were therefore assessed in half of the subjects, whereas changes in forward walking were assessed in the remainder.

\section{Method}

Subjects. A total of 26 Swarthmore undergraduates participated in this experiment in exchange for payment. Subjects were selected without 


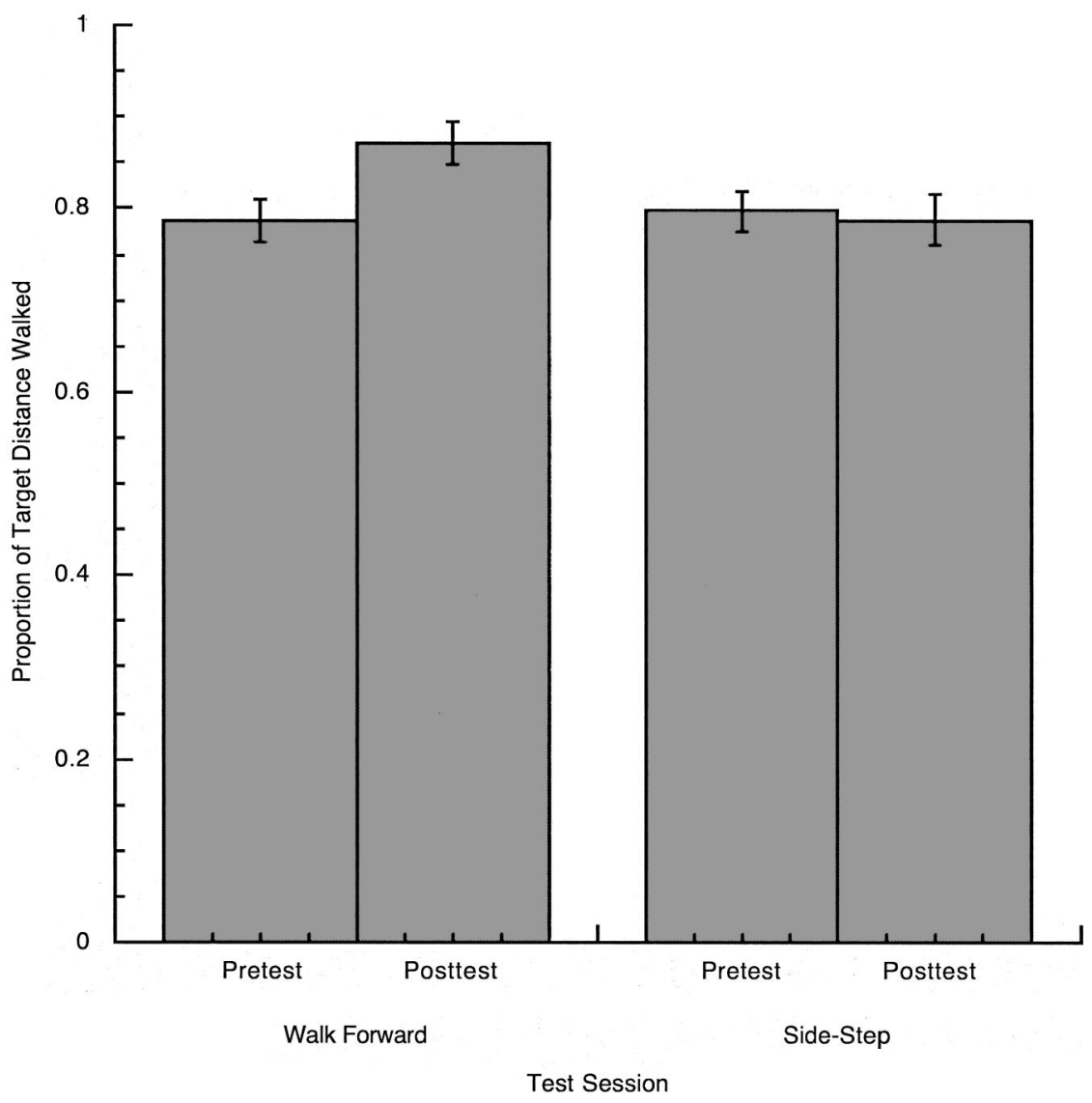

Figure 6. Mean distances walked before and after adaptation (1 min of forward running) for the forward walking and sidestepping conditions of Experiment 4. Error bars represent standard errors, plotted with respect to within-subject differences.

regard to sex, and all had normal or corrected-to-normal vision. They were assigned at random to one of the two experimental conditions.

Design and procedure. All subjects were adapted to forward treadmill running at $9.1 \mathrm{~km} / \mathrm{h}$ for $5 \mathrm{~min}$. Half were tested before and after adaptation with a single trial of forward walking to a target; the other half were tested before and after with sidestepping to a target. The apparatus was the same as in Experiment 4. As in previous experiments, blindfolds and earplugs were worn throughout, and subjects walked using a guidewire (holding a sliding plastic tube) to minimize veering. Target distances were randomly selected from the range of $7-9 \mathrm{~m}$.

\section{Results}

Mean proportions of target distance walked are plotted in Figure 7 as a function of session (pre- or postadaptation) and manner of walking (forward or sidestepping). Adaptation effects were far more variable on sidestepping than on forward running. Seven of the 13 sidestepping subjects showed positive aftereffects, and 6 showed negative aftereffects, but the overall mean change for sidestepping (9\%) was quite similar to the $9 \%$ reported by Rieser et al. (1995). Because of high variability, even a one-tailed test indicated that the proportional distance sidestepped after adaptation $(1.00 \pm 0.06)$ were only marginally higher than those before adaptation $(0.92 \pm 0.05), t(12)=1.63, p=.064$ (one-tailed).
Conversely, whereas the postadaptation distances in the forward-walking condition $(1.08 \pm .06)$ were highly reliably longer than those prior to adaptation $(0.92 \pm 0.04), t(12)=3.70$, $p<.01$, these changes in forward walking $(16 \%)$ were not reliably different from those measured for sidestepping (9\%). Note, however, that these average change values are exactly what might have been predicted on the basis of the totality of Rieser et al.'s (1995) data.

\section{Discussion}

Although the present data do not statistically justify concluding that aftereffects are less strong for sidestepping than for forward walking, neither do they strictly justify the conclusion that there was any transfer at all. Taken together with the findings of Experiment 4 (in which no transfer to sidestepping was found after 1 min of adaptation) and the results reported by Rieser et al. (1995; indicating $9 \%$ overshoot for sidestepping), the conclusion that there was partial transfer seems better justified than the alternative conclusions that there was no transfer or that there was complete transfer.

Partial transfer, however, is still consistent with organismic recalibration. Indeed, in the case of the sidestepping results of 


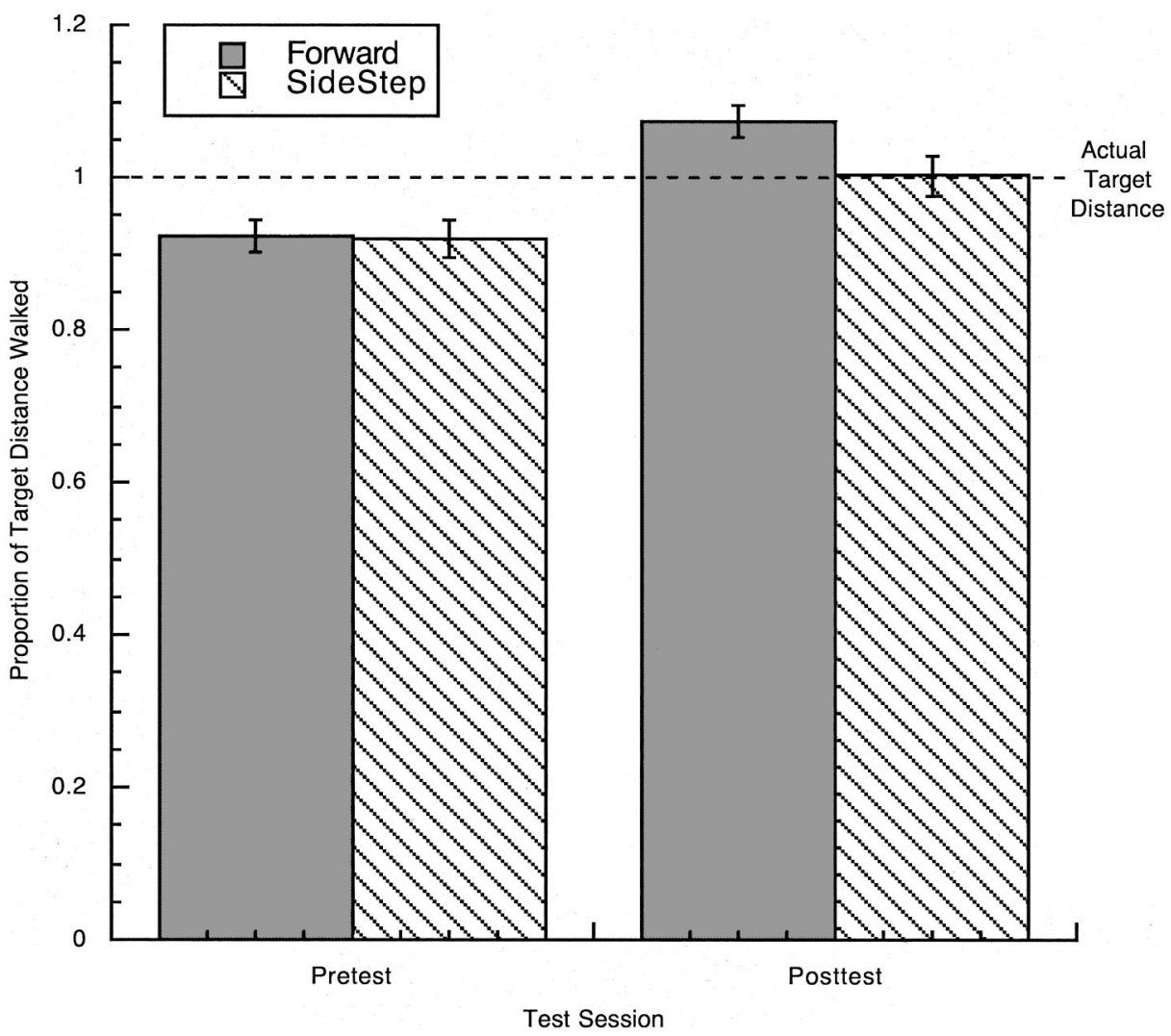

Figure 7. Mean distances walked before and after adaptation (5 min of forward running) for the forward walking and sidestepping conditions of Experiment 5. Error bars represent standard errors, plotted with respect to within-subject differences.

Rieser et al. (1995), it remains possible that lateral organismic recalibration resulted from adaptation to unusual lateral forces required for subjects to maintain position on the treadmill as the treadmill was taken on turns around the parking lot. That is, because Rieser et al.'s treadmill was taken on a route involving turns, there were, in fact, lateral acceleration components during their adaptation procedures, and these might have played a role in producing the recalibration of sidestepping that they measured. Such lateral acceleration events did not occur in the present experiment because the treadmill was on solid ground. Nonetheless, it is possible that lateral drift corrections were occasionally required for those running on the treadmill for the 5-min period of adaptation.

\section{Experiment 6: Short-Term Buildup of the Overshoot Effect}

We have suggested that the overshoot effect rapidly reaches a ceiling of about $15 \%-17 \%$ increase on the basis of evidence that there is little difference in the amount of overshoot whether 1,5 , or even $8 \mathrm{~min}$ of adaptation are used (see Table 1). Here, we sought to measure how much aftereffect was established over short time periods of treadmill running and how much it could be incremented by repeated, rather than extended, adaptation. In this experiment, as in Experiment 5, subjects were adapted for a total of $5 \mathrm{~min}$ of treadmill running, but in this experiment, the adaptation was broken into five increasing segments of time, of which the first was $20 \mathrm{~s}$ and the last was $100 \mathrm{~s}$. Moreover, in this experiment, subjects were then given a rest and retested 5 min later to see if there would be a residual change in walking calibration. Although this experiment raised additional questions that we are not able to resolve in the present article, we include the data to illustrate that (a) very brief periods of adaptation produce fairly large effects, and (b) repeated adaptation may produce larger effects than does extended adaptation.

\section{Method}

Subjects. A total of 16 Swarthmore undergraduates participated in this experiment in exchange for payment. Subjects were selected without regard to sex, and all had normal or corrected-to-normal vision.

Design and procedure. All subjects were adapted to consecutive, increasing periods of treadmill running $(20,40,60,80$, and $100 \mathrm{~s})$. All were pretested with a single walking-to-previewed-target task, with a distance selected randomly from the range of 7-9 $\mathrm{m}$, and then retested with one trial after each period of adaptation before returning (immediately) to the treadmill. Blindfolds and earplugs were worn throughout, with vision only allowed while subjects previewed each target. At the conclusion of this procedure, the blindfold was removed, and subjects were allowed to rest in an adjoining room for $5 \mathrm{~min}$. At the conclusion of the rest period, they were reblindfolded and performed a final walking trial. 


\section{Results}

The average results for each test are shown in Figure 8. Relative to the initial baseline, subjects showed overshoot at every other test. After $20 \mathrm{~s}$ of running, distances walked were (proportionally) $12 \%$ greater than baseline, which was a reliable difference, $t(15)=$ $3.03, p<.01$. After an additional $40 \mathrm{~s}$ of adaptation, distances walked increased again to a total of $18 \%$ more than baseline, which was marginally greater than the distances after the first $20 \mathrm{~s}$ of adaptation, $t(15)=1.98, p=.07$. After an additional $60 \mathrm{~s}$ (for a total of $2 \mathrm{~min}$ ), overshoot (17\%) was still not reliably greater than it was after $20 \mathrm{~s}, t(15)=1.51, p>.10$. It was only after the fourth and fifth adaptation periods (for totals of 200 and $300 \mathrm{~s}$, respectively) that overshoot ( $22 \%$ and $21 \%$ relative to baseline, respectively) was reliably greater than that after only $20 \mathrm{~s}$ of adaptation, $t(15)=3.70, p<.01$, and, $t(15)=2.45, p<.05$, respectively. None of the measurements for adaptations beyond $20 \mathrm{~s}$ differed reliably from each other. This is not to say that there was no increase with increasing adaptation time, only that it was not statistically detectable in the present data. There was clearly a trend for greater overshoot as time increased, but that trend was not reliable, amounting to an increase of only $3 \%$ between the conclusion of the $1 \mathrm{st} \min$ of adaptation and the 5th. Moreover, although the total overshoot after $5 \mathrm{~min}$ had a lower confidence bound of $13 \%$, even after 5 min there was a residual aftereffect of 9\% overshoot, which was also reliably greater than baseline, $t(15)=3.03, p<.01$. Relative to this final baseline, the peak overshoot achieved after 5 min of adaptation represented only a $12 \%$ increase in walking distance.

\section{Discussion}

The idea that adaptation quickly reaches a ceiling is supported by the present results insofar as no reliable increase in adaptation was found between 1 and $5 \mathrm{~min}$, and even $20 \mathrm{~s}$ was enough to provide a shift in calibration (12\%) nearly as large as that found in Experiment 5 after 5 straight minutes of adaptation (16\%). Although the final numbers $(22 \%$ and $21 \%$ overshoot after the fourth and fifth adaptation periods, respectively) suggest that repeated adaptation may provide a stronger aftereffect, it is also possible that the baseline against which these numbers should be evaluated shifted, as is suggested by the overshoot recorded after the 5-min break.

Because of the method of repeated testing, subjects in this study would have been exposed to cue conflict between vestibular (or other inertial) cues and motor estimates of self-motion on each of the adapted test trials. That is, if a subject's motor system specified a distance of $8 \mathrm{~m}$ (the intended distance), whereas his or her other

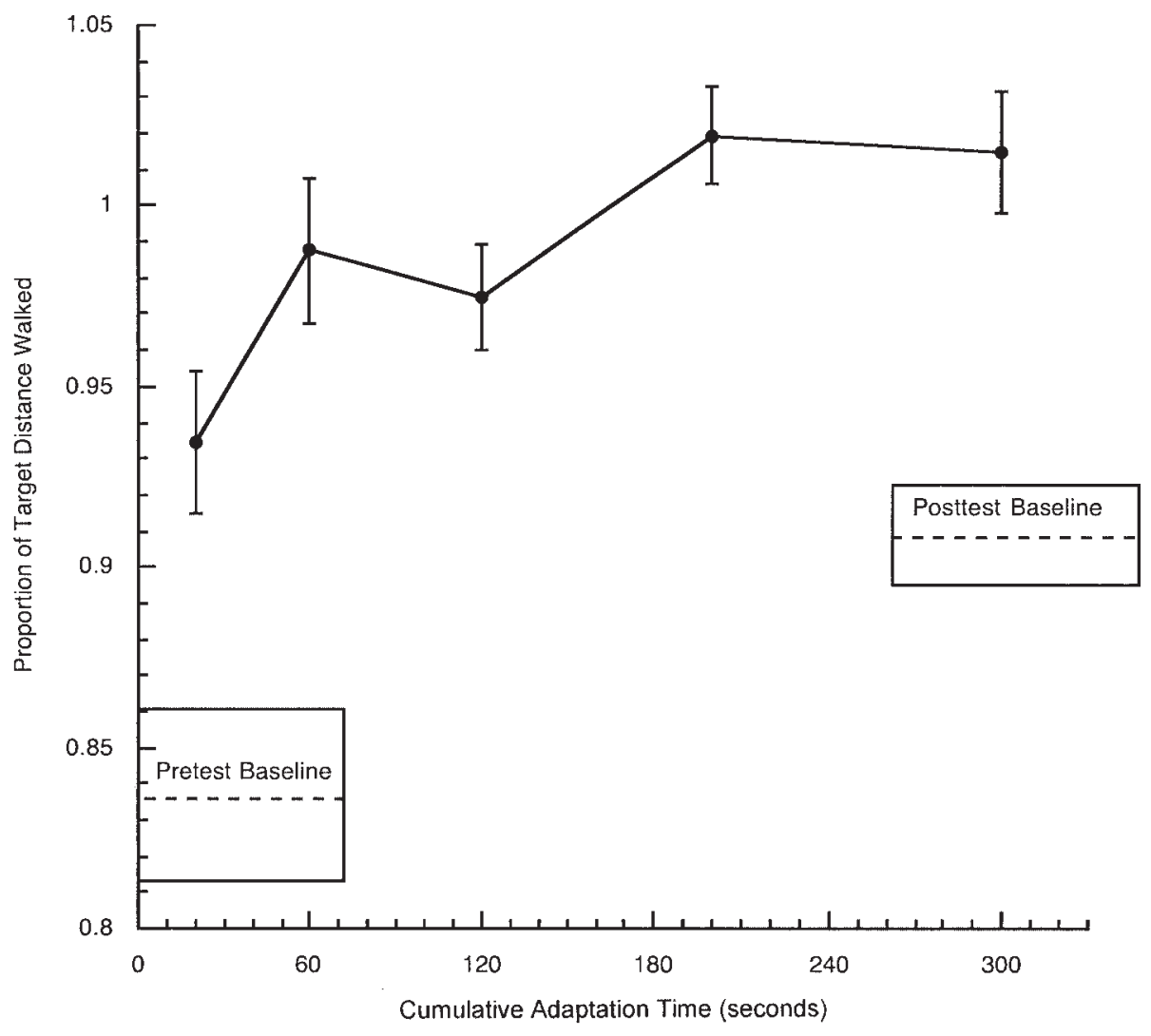

Figure 8. Mean distances walked as a function of cumulative adaptation time in Experiment 6 . The pretest baseline shows the mean for the pretest walking trial plus or minus 1 standard error. The posttest baseline shows the mean plus or minus 1 standard error for the final walking trial after 5 min of rest. Error bars represent standard errors, plotted with respect to within-subject differences. 
systems experienced something more like $9 \mathrm{~m}$ (the actual distance walked), those other systems might have tended to adjust their later estimates downward. Although the conflict would have been fairly slight, it may have contributed to partial adaptation of inertial signals. If this caused these other estimates to be adjusted slightly downward on subsequent trials, that would be sufficient to account for increasing aftereffects in terms of changes in other self-motion estimators. It is thus possible that the "ceiling" was raised slightly by adaptation of other cues to self-motion produced by the continued conflict between these other self-motion signals and motor signals at each testing point (see Ellard \& Shaughnessy, 2003).

This is, of course, quite speculative given that the present data do not strictly justify arguing that any further change occurred after the 1st min of adaptation. Nonetheless, we cannot rule out the possibility that further adaptation (with tests interspersed) might have continued to raise the overshoot. Note that such increases would not contradict the idea that the ceiling on aftereffects found for continuous adaptation (supported by Table 1) may have been due to cue conflict with unadapted cues. Repeated adaptation with testing may produce greater shifts because ancillary cues are themselves recalibrated a bit on each test trial.

\section{Interlude}

We have shown that Anstis's (1995) adaptation procedure (treadmill running with closed eyes) is sufficient to produce Rieser et al.'s (1995) overshoot effect and that the effect transfers only weakly to sidestepping. On the basis of the logic used by Rieser et al. and by Anstis, the fact that overshoot effects are equally large with or without vision ought to be grounds for understanding them as purely motoric. On the one hand, we would now point out that measured effect size (for overshoot, at least) may be intrinsically limited for continuous adaptation, making quantitative comparisons complicated. On the other hand, it has been previously demonstrated that the very logic of equating perception with vision is faulty (Durgin et al., 2003; Durgin \& Pelah, 1999). In particular, drift aftereffects can be generated by adaptation to locomotor activity (solid-ground running or hopping) with closed eyes even when the same motor activity does not produce them when the eyes are open. However, the above-cited experiments were not conducted on treadmills.

Rieser et al. (1995) found an undershoot effect after adaptation to real forward motion that was faster than concurrent treadmill speed during adaptation. That is, Rieser et al. did show compelling evidence that perceptual information influenced the adaptation to treadmill locomotion, though only in their first experiment (their only other example of undershoot, in their Experiment 5, appeared to be much weaker and quite transient-perhaps because the environmental speeds were much lower during adaptation than they had been in their first experiment).

In principle, this perceptuomotor account ought to generalize to the drift effect following treadmill adaptation, but this has never been directly tested. On the basis of the differences between drift and overshoot evidenced by the results of Experiments 2 and 3, we decided to test whether drift effects following adaptation to treadmill locomotion could be reduced if we supplied real environmental motion signals in the manner that Rieser et al. (1995) did.
Experiment 7: Anstis's (1995) Aftereffect Is Moderated by Rieser et al.'s (1995) Adaptation Procedure

Rieser et al.'s (1995) demonstration of undershoot after supply of fast environmental motion $(17 \mathrm{~km} / \mathrm{h})$ compared with locomotor (treadmill) speed $(7 \mathrm{~km} / \mathrm{h}$ ) demonstrates that perceived self-motion is relevant to the effect and that adaptation to treadmill locomotion is not simply motoric. However, most experiments addressing perceptual influences on the drift effect have not used treadmills (e.g., Durgin \& Pelah, 1999). The exception to this is a walking analogue of the drift aftereffect. Durgin et al. (2000; see also Proffitt et al., 2003) adapted subjects to walking on a treadmill and then showed that they inadvertently drifted forward when trying to walk in place without visual feedback. When the subjects were supplied with visual flow in a head-mounted display during adaptation, these walking drift aftereffects were somewhat reduced. However, in those experiments, the subjects were required to look to the side as they walked on the treadmill. This unusual reorientation of the head with respect to the torso may have also played a role in reducing adaptation.

Having established that the overshoot aftereffect can be produced in the same way as the drift aftereffect, we sought to determine to what extent the drift aftereffect could be moderated by the technique of supplying real forward movement during adaptation that was actually faster than the speed of the treadmill belt. Rieser et al. (1995) did this by towing their treadmill at 17 $\mathrm{km} / \mathrm{h}$. Although we knew that during adaptation, our towed subjects would still be controlling their actions with respect to the reference frame of the treadmill, we predicted that providing real forward movement during treadmill running would reduce or reverse the drift aftereffect. Unfortunately, because the motor on our towing device was unable to attain the desired speed except when driven down a slight grade, we were only able to supply 1 min of continuous adaptation. However, we expected even this amount to be sufficient to reduce the drift effect.

\section{Method}

Subjects. A total of 12 Swarthmore undergraduates participated in this experiment in exchange for payment. Subjects were selected without regard to sex, and all had normal or corrected-to-normal vision.

Apparatus. Our Landice treadmill was mounted on a wooden trailer with pneumatic tires. This trailer was hitched to the back of an electric golf cart that carried a generator used to power the treadmill. A chest-high safety cage was built around the treadmill to prevent subjects from falling off the trailer while the golf cart was in motion. Subjects were also given a protective helmet to wear throughout the experiment. A downhill course along a campus road was used, which could be traversed by this golf cart apparatus in $1 \mathrm{~min}$ at an average speed of $17 \mathrm{~km} / \mathrm{h}$.

RIP task. Before each RIP assessment, individual subjects secured a blindfold around their eyes. They then attempted to run in place for $20 \mathrm{~s}$, timed by an experimenter. The amount of inadvertent forward drift during this period was measured in centimeters and divided by $20 \mathrm{~s}$ to produce an average velocity of forward drift.

To ensure quick and easy blindfolding before each RIP assessment, subjects wore the blindfold throughout the experiment, pushing it up on their foreheads when it was not needed. Subjects also wore foam earplugs throughout the experiment to minimize usage of auditory localization information during testing.

Adaptation. Each subject experienced three different kinds of adaptation, each of a 1-min duration. During the treadmill-stationary adaptation, 
subjects jogged on the treadmill at $7 \mathrm{~km} / \mathrm{h}$ without being towed by the golf cart. During the treadmill-moving adaptation, subjects jogged on the treadmill at $7 \mathrm{~km} / \mathrm{h}$ while being towed by the golf cart at $17 \mathrm{~km} / \mathrm{h}$. During the passive-moving adaptation, subjects stood on the treadmill while being towed by the golf cart at $17 \mathrm{~km} / \mathrm{h}$. Subjects were not blindfolded during any of the adaptation periods.

Design and procedure. Baseline performance on the RIP task was assessed at the beginning of the experiment, and performance was reassessed following each adaptation period. All subjects completed three different adaptation periods, but the order in which these were completed varied. The order of the adaptation periods was always such that the passive-moving adaptation preceded the treadmill-moving adaptation so that subjects could become comfortable with riding on the golf cart apparatus before they were required to run on the treadmill concurrently.

The entire experimental procedure and safety precautions were described to subjects before the experiment began. Half of the subjects then experienced the procedure as follows: (a) baseline RIP assessment, (b) treadmill-stationary adaptation and posttest RIP assessment, (c) passivemoving adaptation and posttest RIP assessment, (d) return to starting point, and (e) treadmill-moving adaptation and posttest RIP assessment. The other half of the subjects experienced the procedure slightly differently: (a) baseline RIP assessment, (b) passive-moving adaptation and posttest RIP assessment, (c) return to starting point, (d) treadmill-moving adaptation and posttest RIP assessment, (e) return to starting point, (f) second baseline RIP assessment, and $(\mathrm{g})$ treadmill-stationary adaptation and posttest RIP assessment. Note that only in the second version of the procedure was a second baseline RIP assessment made. This design aspect was intended to mimic the fact that in the first procedure the passive-moving adaptation intervened between the two treadmill conditions.

\section{Results}

As expected, subjects showed reliable forward drift during the initial pretest, with an average velocity of $8.9 \mathrm{~cm} / \mathrm{s}, t(11)=7.87$, $p<.01$. Aftereffects for each adaptation condition were calculated as changes in the average velocity of inadvertent advance relative to the initial pretest. As shown in Figure 9, the increase in drift velocity was much stronger following the treadmill-stationary condition $(+7.7 \mathrm{~cm} / \mathrm{s})$ than following the treadmill-moving condition $(+3.2 \mathrm{~cm} / \mathrm{s}), t(11)=3.00, p<.05$. Nonetheless, both of these values represent reliable increases from baseline in average velocity of inadvertent drift, $t(11)=6.41, p<.01$, and, $t(11)=2.92$, $p<.05$, respectively. Merely riding down the hill without running (the passive-moving condition) actually reduced the amount of drift by $1.9 \mathrm{~cm} / \mathrm{s}, t(11)=2.87, p<.05$. If the decreased drift from merely riding on the treadmill is subtracted from the increased drift from running on the stationary treadmill, the result is still $2.6 \mathrm{~cm} / \mathrm{s}$ greater than the drift after riding and running concurrently, $t(11)=$ $1.84, p<.05$ (one-tailed)

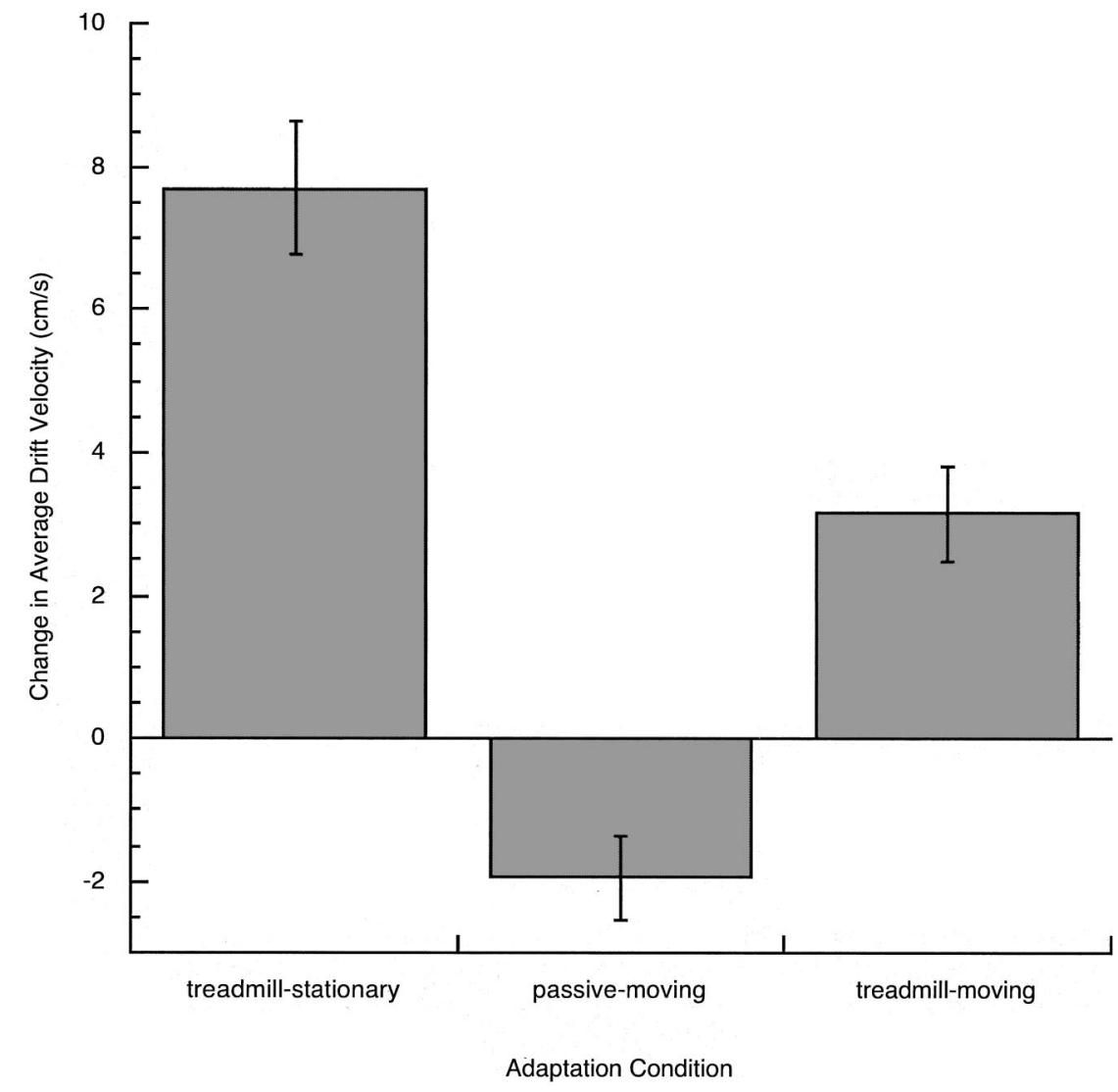

Figure 9. Changes in velocity of inadvertent forward drift by condition in Experiment 7. Error bars represent standard errors, plotted with respect to within-subject differences. 


\section{Discussion}

As predicted, the Rieser et al. (1995) technique for supplying real forward movement during adaptation to treadmill locomotion did in fact dampen the drift aftereffect. This confirms that the drift aftereffect, like the overshoot effect, can be affected by concurrent movement through the environment, even when adaptation is conducted on a treadmill.

Although the size of this change was less than might have been expected, we also know that Rieser et al.'s (1995) measurements of undershoot were much weaker than their measurements of overshoot and possibly more transient (see Rieser et al., 1995, Experiment 5). If, during the treadmill-moving adaptation period, our subjects had truly experienced themselves as moving faster than normal as a result of their motor activity, they ought, perhaps, to have actually drifted less after the treadmill-moving adaptation than before it (just as subjects in the Rieser et al., 1995, movingfaster-than-walking condition undershot targets during blind walking). However, our adaptation period was much shorter than Rieser et al.'s ( 1 min vs. $8 \mathrm{~min}$ ), and in retrospect, it appears likely that subjects were probably more focused on maintaining position on the treadmill than on the passing scenery during the initial minute than they might have been during a longer period of adaptation. To the extent that subjects' primary awareness was on maintaining stasis with respect to the treadmill, we would expect this condition to produce results similar to running on a stationary treadmill. The main purpose of the experiment was fulfilled, nonetheless, because the drift aftereffect was indeed reduced by the addition of real movement through the environment during treadmill adaptation.

\section{General Discussion}

The seven experiments presented here support the conclusion that adaptation to treadmill locomotion entails perceptuomotor cue conflict, even when the eyes are closed, and that the resulting recalibrations of motor activity generalize to different forward locomotor tasks, including walking to a target and running in place, while showing limited transfer to sidestepping. These patterns of generalization are more consistent with classic organismic recalibration than with environment-based or goal-specific recalibration.

Experiments 1, 2, and 4 showed that marked overshoot on the task of walking without visual feedback to a visually previewed target can be induced by 1 min of treadmill jogging. Indeed, this short period of adaptation produced forward overshoot effects as strong as those reported by Rieser et al. (1995) after 8 min of treadmill locomotion while being carried through the environment. This rapid adaptation was shown to occur in as little as $20 \mathrm{~s}$ in Experiment 6.

In Experiment 4, however, we found that $1 \mathrm{~min}$ of adaptation to forward treadmill running was insufficient to produce a change in sidestepping to a target. Moreover, in Experiment 5 we found that even $5 \mathrm{~min}$ of adaptation produced only a weak effect, similar in magnitude to that reported by Rieser et al. (1995). These findings support the notion that adaptation effects are organized primarily not around specific goals (e.g., locomotion in general) but, rather, around the organism's evaluation of its own locomotor activities (e.g., forward self-motion associated with specific axes of locomotor movement).
Experiment 3 characterized the drift effect as involving a relatively steady rate of inadvertent forward advance, whereas Experiment 7 showed that the amount of drift aftereffect depends not only on motor activity but also on the perceptual information available during adaptation (see also Durgin et al., 2003; Durgin \& Pelah, 1999).

Although there are clear similarities between the drift and overshoot effects, and although both can be produced by the same adaptation procedure, there are also differences between them. A chief difference is that there appears to be a ceiling on the amount of overshoot that can be generated by adaptation to treadmill locomotion such that $5 \mathrm{~min}$ of adaptation produces no more overshoot than does $1 \mathrm{~min}$. Overshoot in both cases is $16 \%-17 \%$. In contrast, the drift effect is measurably stronger (by a factor of 2) after 2 min than after $1 \mathrm{~min}$, showing an additional $10 \mathrm{~cm} / \mathrm{s}$ after $2 \mathrm{~min}$ but only an additional $5 \mathrm{~cm} / \mathrm{s}$ after $1 \mathrm{~min}$.

\section{Modeling the Ceiling Effect}

There are several types of explanations as to why overshoot shows a ceiling whereas drift does not. At least one of these types of explanations is consistent with the notion that the motor estimates of self-motion are altered identically in both cases, whereas the extent of adaptation in the case of overshoot is masked by other, task-specific information. For example, suppose that walking to a target without vision involves both motor estimates of self-motion and vestibular inputs (or the integration of inertial signals more generally; see Mittelstaedt \& Mittelstaedt, 1996) concerning self-motion. This is a reasonable supposition, because it is known that subjects can make judgments about self-motion from inertial signals during passive self-motion (Harris et al., 2000; Israël \& Berthoz, 1989), even if those judgments seem to partly reflect default speeds of motion (Mittelstaedt \& Mittelstaedt, 2001). Because inertial information concerning self-motion is not put into conflict with perceived self-motion during treadmill adaptation (people do not have the illusion that they are moving when running on a treadmill, nor do their inertial systems experience any net acceleration), inertial signals should not be recalibrated by treadmill running the way that motor signals are. Indeed, it is even possible that inertial signals are recalibrated in the opposite direction-toward higher estimates of self-motion speed-because of cue conflict with the motor estimates during treadmill running.

Depending on how inertial information is integrated with motor estimates of self-motion, the net result of combining adapted motor estimates and unadapted (or oppositely adapted) inertial estimates might easily produce an apparent ceiling on the expression of motor recalibration in the form of overshoot. In essence, the true value of the underlying motor estimate might be partly masked by combination with an increasingly disparate inertial (e.g., vestibular) estimate of self-motion. The apparent ceiling on the overshoot effect might thus represent the lower bound of the vestibular (or other) estimate of the distance of self-motion.

This explanation predicts that the ceiling on the overshoot effect would not apply to the drift effect, because inertial signals concerning the absence of self-motion are of limited value in controlling stationary running. That is, remaining stationary while running in place demands that one produce no net acceleration. But the small net accelerations involved during inadvertent drift (e.g., 
accelerating to even as much as $15 \mathrm{~cm} / \mathrm{s}$ over 3 to $4 \mathrm{~s}$ ) are likely to be more difficult to detect (not perceptually different from zero) and more swamped by noise than are the net accelerations involved in actual forward walking. Studies of vestibuloocular reflex during walking in place indicate that small head rotations are well compensated for by gaze (Grossman, Leigh, Bruce, Huebner, \& Lanska, 1989), so it is not impossible that concomitant linear accelerations are also detectable. However, the functionality of gaze stability during walking is clear. What is unclear is how precise the net integration of small otolith signals can be. The fact that people (a) inadvertently drift when attempting to run in place even before adaptation and (b) cannot maintain position on a treadmill of constant velocity without some noninertial form of perceptual feedback is evidence that the integration of forward inertial signals during stationary running is likely quite difficult and could be expected to play little role in curtailing gradual drift.

A somewhat different type of explanation of the ceiling on overshoot could be that locomotor estimates of self-motion during walking are so well-learned (and result from the confluence of so many sensory factors available even with closed eyes, including bounce and sway, haptic force detection during footfalls, etc.) that there is a great deal of calibrational inertia associated with walking, whereas running in place is a fairly unusual task and, therefore, more susceptible to adaptive recalibration.

Finally, we have also discussed the possibility that drift showed no ceiling following treadmill adaptation because the adaptation and testing tasks were so similar: to run so as to not move relative to some reference frame. Although there may be some truth to this, the fact that drift was reduced by supplying environment-relative self-motion in Experiment 7 indicates that drift is also sensitive to what actually unfolds during treadmill locomotion (e.g., concomitant environmental motion), not simply to the intended goals of that locomotion (to stay on the treadmill).

\section{Models of Perceptuomotor Organization}

Human locomotion systems are highly skilled and adaptive. These systems must maintain calibration in response to changing physiological and environmental conditions. Rieser et al. (1995) concluded that the overshoot aftereffect generalized on the basis of action goals because they found that adaptation to walking on their mobile treadmill apparatus transferred to sidestepping but not to throwing. However, the present evidence suggests that such transfer is relatively weak. Moreover, we have shown that two activities with very different functional goals (running in place vs. walking to a target) both seem to reflect the same underlying adaptations to locomotor activity with enhanced or absent optic flow. This result is more consistent with a change in effector-specific (organismic) calibration than with a goal-specific recalibration or environmental recalibration. A similar point can be made from previous work on the absence of interlimb transfer in hopping versions of Anstis's (1995) and Rieser et al.'s (1995) aftereffects (Durgin et al., 2003). We do not think that Rieser et al.'s idea of a functional organization of recalibration should be completely abandoned, but we think Rieser et al. dismissed organismic recalibration on insufficient evidence. We assume, for example, that the overlap in overshoot and drift aftereffects may indeed arise from the recalibration of self-motion perception, a functional system of the sort Gibson (1966) might have favored. However, we think that the recalibra- tion of kinesthetic estimates of self-motion (whether in the form of motor predictions or proprioceptive feedback) is mainly responsible for the drift and overshoot effects that have been reported and that these recalibrations, although driven by perceptual feedback during adaptation, are effector specific (Durgin et al., 2003).

We should note that although there is a conceptual analogy between the task of walking to a previewed target and that of running in place (i.e., the latter can be construed as running to the location where one currently is standing), there is also a strong disanalogy. Specifically, whereas the walking task requires that one stop when one gets to the target (it is defined spatially), the task of running in place is defined as maintaining zero velocity for a specific period of time (it is defined temporally). Temporal integration has been implicated in studies of vestibular estimation of self-motion (Mittelstaedt \& Mittelstaedt, 2001), in which it has appeared that travel time itself is used as a measure of distance traveled. Obviously, such temporal strategies are irrelevant to stationary running, in which temporal limits are imposed by the experimenter, but they could play a role in walking to a target.

\section{Against the Hegemony of Vision}

Prior emphases on exclusively visual information about selfmotion in locomotor recalibration were simply mistaken. Some of these biases may have resulted from the work of Lishman and Lee (1973), who demonstrated visual capture of self-motion perception when visual and vestibular information were set in conflict. In this article, we have presented evidence that the nonvisual perception of self-motion while walking and while attempting stationary running can be altered by a variety of perceptuomotor experiences, including some that involve vision (Experiment 7) and some that do not (Experiments 1-6). Although perceptual self-motion estimates in these particular tasks may draw on different subsets of the available sources of information concerning self-motion, it seems likely that a common site of recalibration is in motor predictions and kinesthetic proprioception (which were always linked in our experiments). Treadmill locomotion, with or without visual input, will almost always involve conflict between motor-kinesthetic activity and other sources of information specifying self-motion. This conflict has consequences for locomotor calibration in tasks in which the goal is to remain stationary as well as for those with a distant locomotor target.

Unfortunately, the claim that visual flow is necessary to and sufficient for locomotor recalibration seems to have been widely assumed. For example, Withagen and Michaels (2002), who claimed to support the general picture painted by Rieser et al. (1995), had people walk on a treadmill while in a CAVE (cave automatic virtual environment) - a room with a three-wall virtual display - that simulated visual speeds faster or slower than the treadmill walking speeds they used. Although Withagen and Michaels's results seem to show that adaptation transferred partially to crawling (which does, after all, still involve the legs), the only changes that they report show overshoot for both walking and crawling (even in the condition in which simulated visual speed was too fast and they should have observed undershoot!). However, because their absolute treadmill speed differed by a factor of 2 between their two adaptation conditions, the differences they did find between the conditions could all be accounted for on the basis of treadmill speed alone. When dismissing this interpretation of 
their data, Withagen and Michaels cited Rieser et al.'s demonstration that biomechanical activity alone (i.e., activity with closed eyes) cannot account for overshoot. As we have shown here, that argument no longer appears valid.

Although we agree with those who suppose that the perception of self-motion is important to development of the overshoot aftereffect, we also contend that self-motion perception is not limited to vision, which seems to have to compete with other senses when they are in conflict. Withagen and Michaels's (2002) results, in which overshoot is simply larger after walking on a treadmill for $15 \mathrm{~min}$ at $6 \mathrm{~km} / \mathrm{h}$ than it is at $3 \mathrm{~km} / \mathrm{h}$, are not good evidence that the CAVE played a role in moderating the aftereffect. Durgin and Pelah (1999) reported that supplying artificial optical flow to treadmill runners by means of a single $\left(120^{\circ}\right.$ wide $)$ wall display of expanding visual flow had no influence on the amount of resulting drift. Durgin and Pelah also noted that subjects' perceptual experience relating to self-motion throughout the adaptation period was that they were running without actually going anywhere.

Conversely, Anstis (personal communication, cited in Durgin \& Pelah, 1999) found that congenitally blind subjects showed drift aftereffects after walking on a treadmill. He hoped to show by this that because the faculty of vision was clearly irrelevant for these subjects, the effects must be motoric or postural. As we have emphasized, however, this is a false dichotomy. Blind individuals on a treadmill are controlling their self-motion with respect to the treadmill using perceptual feedback (e.g., haptic contact with the handrails). That is, Anstis's subjects also were experiencing an absence of self-motion while their legs moved on the treadmill, and it was this conflict, not the treadmill per se, that we believe produced the change in calibration.

In the literature on podokinetic afterrotation (PKAR), the opposite conclusion has been argued, but the paradigms defining PKAR are quite different, and posture may be more relevant to rotation. Gordon et al. (1995) reported that extended walking on the perimeter of a circular disk produced circular paths later when subjects attempted to walk straight without visual feedback. The inner leg during adaptation travels the shorter path, both during adaptation and (inadvertently) during testing. This is consistent with the differential calibration of the two legs, such as occurs after hopping on a treadmill (Durgin et al., 2003). Intriguingly, however, attempts to walk backward without vision (after adaptation to forward walking in a circle) produce oppositely curved paths, with the "inner" leg going on the farther path (Earhart et al., 2001). This suggests that PKAR may represent a renormalization of a legtorso orientation rather than simply of the linear perceived gain of the legs. An alternative paradigm for generating and measuring similar rotary aftereffects has subjects step in place on a rotating disk such that they stand over the axis of rotation (Weber, Fletcher, Gordon, Melvill Jones, \& Block, 1998). Unlike in the earlier paradigm, the two legs are now actually applying force in different linear directions during adaptation. This also produces curved paths when subjects later attempt to walk straight and inadvertent rotations when they try to step in place, and it has also been associated with pelvic rotation (Earhart et al., 2002). Jürgens, Boß, and Becker (1999) have reported that these latter effects are indifferent to the presence of visual feedback provided during adaptation, and they have argued that the effects are therefore due not to sensory conflict but to postural adaptation. This postural interpretation may be correct in the case of PKAR, but we hope that visual information will not continue to be considered the entirety of self-motion perception. Posture, too, is a matter of kinesthetic perception.

\section{Rapid Calibration}

Rieser et al. (1995) concluded that their results showed that "human adults rapidly adjust the calibration of their walking" (p. 496), but as our experiments have shown, the adjustment is much more rapid ( $20 \mathrm{~s}$ is plenty of time to produce strong overshoot) than Rieser et al. realized. This very rapid time scale is actually much more in line with one of the nonexperimental questions that Rieser et al. sought to understand: How can a backpacker adjust the strength of jumping across a stream, given that he or she must take footing into account as well as the load of the backpack. In our experience, the question of footing is assessed perceptually by testing the ground with the foot a bit before launching-which essentially allows for a few seconds of "adaptation" to the amount of force that, for example, sandy or muddy ground might absorb. This seems like an excellent example of nonvisual information playing a role in motor adjustments that are based on (haptic) perceptual feedback derived from exploratory behavior. One reviewer suggested that this might be described as assessment of the affordance of the surface in question, but it should be emphasized that the assessment is not only of whether the surface affords sufficient support but also of how much force is appropriate for the surface. In any case, these kinds of motor adjustments need to be extremely rapid to be useful.

Given the preliminary evidence presented here that average overshoot quickly reaches a ceiling at about $17 \%$, one wonders whether this ceiling is somehow reflective of the range of motor gains encountered in everyday life during walking on typical surfaces. Since limbs do change over time, presumably short-term recalibrations can become longer term ones with prolonged exposure over the course of daily life. The absolute range of change would almost certainly be greater if a person was repeatedly adapted to treadmill activity without any reexposure to normal walking.

However, we think that the aftereffect would be maximized if all of the interoceptive signals available during normal walking (including vestibular information and kinesthetic proprioception) were present during adaptation and only external perceptual information was altered. Indeed, using a wide-area virtual reality system in which visual feedback to normal walking locomotion was altered, Durgin, Fox, Lewis, and Walley (2002) recently found precisely this outcome: changes in walking calibration of $35 \%$ after less than $4 \mathrm{~min}$ of adaptation. Their interpretation was that adaptation to normal walking with altered feedback is a more powerful stimulus for the recalibration of normal walking because inertial signals produced by the physical accelerations through space are also adapted in this case to conflicting visual information.

\section{Perceptuomotor Integration and Calibration}

Although the integration of sensory and motor information about self-motion is not well understood, it is likely that internal models in the brain are multimodal (e.g., Mergner \& Rosemeier, 1998; Wiener, Berthoz, \& Zugaro, 2002). Moreover, there is 
two-way interaction between motor and perceptual information. For example, Pelah and Barlow (1996) showed that adaptation to treadmill running also has the consequence of producing an increase in the perceived speed of visual motion while walking.

It has also been shown that the perception of simulated visual speed is reduced during treadmill locomotion (Pelah, Thurrell, \& Berry, 2002; Thurrell, Pelah, \& Distler, 1998). Durgin, Gigone, and Scott (2005) have recently shown that this visual speed reduction is subtractive (rather than multiplicative) and also occurs during passive self-motion (see also Pavard \& Berthoz, 1977; Wallach \& Flaherty, 1975). Moreover, both active (Pelah \& Boddy, 1998) and passive (Harris, Morgan, \& Still, 1981; Wallach $\&$ Flaherty, 1975) locomotion suppress the motion aftereffect for correlated optic flow. The implications of these various visual consequences of self-motion are not fully understood, but they do not seem to follow simple mathematical separation of retinal motion into self-motion and world motion. For example, Durgin et al. (2005) found no evidence of addition of visual speed of an expanding flow field when subjects were passively moved backward, though there was subtraction of visual speed for a contracting flow field. There is good evidence therefore that these speed reductions cannot be considered specifically the results of motor predictions (e.g., Wolpert \& Flanagan, 2001) because they occur similarly for active treadmill locomotion and for passive selfmotion (Durgin et al., 2005).

Barlow's (1990) theory of subtractive inhibition seems wellsuited to explain many of these phenomena while simultaneously addressing the kinds of adaptation demonstrated in the present experiments. Barlow proposed that subtractive inhibition might develop between sensory signals that are reliably correlated and, therefore, copredictive. Two natural consequences of such a scheme are reduced visual-motion perception while walking (because visual motion is predicted by motor action and other sensory signals specifying self-motion) and a change in the nonvisual perception of self-motion following treadmill adaptation (because the nonvisual perception of self-motion now associated with walking has been altered during adaptation). Although this view departs in emphasis from the idea of an internal model that makes explicit predictions, it is possible to construe Barlow's hypothesized web of intercorrelations among sensory signals as a kind of embodied internal model that allows for both intersensory prediction and intersensory calibration.

\section{Conclusions}

Both drift aftereffects and overshoot aftereffects can be regarded as resulting from recalibrations of nonvisual estimates of selfmotion resulting from conflict between, for example, motor estimates and the perception of self-motion. Prior studies that concluded or assumed that visual self-motion perception was required for locomotor recalibration were incorrect. The experiments reported in this article support the conclusion that locomotor recalibration to treadmill running engages the perception of selfmotion whether the eyes are open or not. Although the integration of various idiothetic and allothetic sources of information about self-motion is not well understood, it is clear that self-motion perception and locomotor recalibration must be understood as involving more than simply the combination of motor action and visual feedback. It appears likely that a multimodal spatial sense of self-motion served as the principal perceptual feedback that recalibrates motor estimates of self-motion in the experiments we have described.

\section{References}

Anstis, S. M. (1995). Aftereffects from jogging. Experimental Brain Research, 103, 476-478.

Barlow, H. B. (1990). A theory about the functional role and synaptic mechanism of visual aftereffects. In C. Blakemore (Ed.), Vision: Coding and efficiency (pp. 363-375). Cambridge, England: Cambridge University Press.

Durgin, F. H. (1996). Visual aftereffect of texture density contingent of color of frame. Perception \& Psychophysics, 58, 207-223.

Durgin, F. H., Banton, T., Walley, K., Proffitt, D. R., Steve, J., \& Lewis, J. (2000). Perceptuomotor recalibration in a virtual world [Abstract]. Investigative Ophthalmology \& Visual Science, 41, S799.

Durgin, F. H., Fox, L. F., \& Kim, D. H. (2003). Not letting the left leg know what the right leg is doing: Limb-specific locomotor adaptation to sensory-cue conflict. Psychological Science, 14, 567-572.

Durgin, F. H., Fox, L. F., Lewis, J., \& Walley, K. A. (2002). Perceptuomotor adaptation: More than meets the eye [Abstract]. Abstracts of the Psychonomic Society, 7, 103-104.

Durgin, F. H., Gigone, K., \& Scott, R. (2005). The perception of visual speed while moving. Journal of Experimental Psychology: Human Perception and Performance, 31, 339-353.

Durgin, F. H., \& Pelah, A. (1999). Visuomotor adaptation without vision? Experimental Brain Research, 127, 12-18.

Durgin, F. H., Pelah, A., \& Amiruddin, S. (1998). Measures of visuomotor adaptation to anomalous optic flow [Abstract]. Investigative Ophthalmology \& Visual Science, 39, S1094.

Earhart, G. M., Melvill Jones, G., Horak, F. B., Block, E. W., Weber, K. D., \& Fletcher, W. A. (2001). Forward versus backward walking: Transfer of podokinetic adaptation. Journal of Neurophysiology, 86, 1666-1670.

Earhart, G. M., Melvill Jones, G., Horak, F. B., Block, E. W., Weber, K. D., \& Fletcher, W. A. (2002). Podokinetic after-rotation following unilateral and bilateral podokinetic stimulation. Journal of Neurophysiology, 87, 1138-1141.

Ellard, C. G., \& Shaughnessy, S. C. (2003). A comparison of visual and nonvisual sensory inputs to walked distance in a blind-walking task. Perception, 32, 567-578.

Gibson, J. J. (1966). The senses considered as perceptual systems. Boston: Houghton Mifflin.

Gordon, C. R., Fletcher, W. A., Melvill Jones, G., \& Block, E. W. (1995). Adaptive plasticity in the control of locomotor trajectory. Experimental Brain Research, 102, 540-545.

Grossman, G. E., Leigh, R. J., Bruce, E. N., Huebner, W. P., \& Lanska, D. J. (1989). Performance of the human vestibuloocular reflex during locomotion. Journal of Neurophysiology, 62, 264-272.

Harris, L. R., Jenkin, M., \& Zikovitz, D. C. (2000). Visual and non-visual cues in the perception of linear self-motion. Experimental Brain Research, 135, 12-21.

Harris, L. R., Morgan, M. J., \& Still, A. W. (1981, September 10). Moving and the motion after-effect. Nature, 293, 139-141.

Israël, I., \& Berthoz, A. (1989). Contribution of the otoliths to the calculation of linear displacement. Journal of Neurophysiology, 62, 247-263.

Israël, I., Grasso, R., Georges-François, P., Tsuzuki, T., \& Berthoz, A. (1997). Spatial memory and path integration studied by self-driven passive linear displacement: I. Basic properties. Journal of Neurophysiology, 77, 3180-3192.

Jürgens, R., Boß, T., \& Becker, W. (1999). Podokinetic after-rotation does not depend on sensory conflict. Experimental Brain Research, 128, 563-567. 
Lishman, J. R., \& Lee, D. N. (1973). The autonomy of visual kinaesthesis. Perception, 2, 287-294.

Loomis, J. M., \& Beall, A. C. (2004). Model-based control of perception/ action. In L. M. Vaino, S. A. Beardsley, \& S. K. Rushton (Eds.), Optic flow and beyond (pp. 421-441). Dordrecht, the Netherlands: Kluwer Academic.

Loomis, J. M., Da Silva, J. A., Fujita, N., \& Fukusima, S. S. (1992). Visual space perception and visually directed action. Journal of Experimental Psychology: Human Perception and Performance, 18, 906-921.

Marlinsky, V. V. (1999). Vestibular and vestibulo-proprioceptive perception of motion in the horizontal plane in blindfolded man-I. Estimations of linear displacement. Neuroscience, 90, 389-394.

Mergner, T., \& Rosemeier, T. (1998). Interaction of vestibular, somatosensory and visual signals for postural control and motion perception under terrestrial and microgravity conditions: A conceptual model. Brain Research Reviews, 28, 118-135.

Mittelstaedt, M.-L., \& Mittelstaedt, H. (1996). The influence of otoliths and somatic graviceptors on angular velocity estimation. Journal of Vestibular Research: Equilibrium \& Orientation, 6, 355-366.

Mittelstaedt, M.-L., \& Mittelstaedt, H. (2001). Idiothetic navigation in humans: Estimations of path length. Experimental Brain Research, 139, $318-332$.

Pavard, B., \& Berthoz, A. (1977). Linear acceleration modifies the perceived velocity of a moving visual scene. Perception, 6, 529-540.

Pelah, A., \& Barlow, H. B. (1996, May 23). Visual illusion from running. Nature, 381, 283.

Pelah, A., \& Boddy, A. (1998). Adaptive modulation of the motion after-effect by walking [Abstract]. Journal of Physiology, 506P, 111P$112 \mathrm{P}$.

Pelah, A., Thurrell, A. E. I., \& Berry, M. (2002). Reduction of perceived visual speed during walking: Evidence against the involvement of attentional or vestibular mechanisms [Abstract]. Journal of Vision, 2, 630a. Retrieved from http://journalofvision.org/2/7/630/

Philbeck, J. W., O'Leary, S., \& Lew, A. L. B. (2004). Large errors, but no depth compression, in walked indications of exocentric extent. Perception \& Psychophysics, 66, 377-391.

Pick, H. L., Jr., Rieser, J. J., Wagner, D., \& Garing, A. E. (1999). The recalibration of rotational locomotion. Journal of Experimental Psychology: Human Perception and Performance, 25, 1179-1188.

Proffitt, D. R., Stefanucci, J., Banton, T., \& Epstein, W. (2003). The role of effort in perceiving distance. Psychological Science, 14, 106-112.

Reynolds, R. F., \& Bronstein, A. M. (2003). The broken escalator phenomenon: Aftereffect of walking onto a moving platform. Experimental Brain Research, 151, 301-308.

Rieser, J. J., Ashmead, D. H., Talor, C. R., \& Youngquist, G. A. (1990). Visual perception and the guidance of locomotion without vision to previously seen targets. Perception, 19, 675-689.
Rieser, J. J., Pick, H. L., Jr., Ashmead, D. H., \& Garing, A. E. (1995). Calibration of human locomotion and models of perceptual-motor organization. Journal of Experimental Psychology: Human Perception and Performance, 21, 480-497.

Riley, M. A., \& Turvey, M. T. (2001). Inertial constraints on limb proprioception are independent of visual calibration. Journal of Experimental Psychology: Human Perception and Performance, 27, 438-455.

Schaffer, E., \& Durgin, F. H. (2005). Visual-vestibular dissociation: Differential sensitivity to acceleration and velocity. Manuscript submitted for publication.

Sun, H. J., Campos, J. L., \& Chan, G. S. W. (2004). Multisensory integration in the estimation of relative path length. Experimental Brain Research, 154, 246-254.

Thomson, J. A. (1983). Is continuous visual monitoring necessary in visually guided locomotion? Journal of Experimental Psychology: Human Perception and Performance, 9, 427-443.

Thurrell, A. E. I., Pelah, A., \& Distler, H. K. (1998). The influence of non-visual signals of walking on the perceived speed of optic flow [Abstract]. Perception, 27, 147-148.

von Holst, E., \& Mittelstaedt, H. (1950). Das Reafferenzprinzip: Wechselwirkungen zwischen Zentralnervensystem und Peripherie [The reafference principle: Reciprocal effects between central nervous system and periphery]. Naturwissenschaften, 37, 464-476.

Wallach, H., \& Flaherty, E. W. (1975). A compensation for field expansion caused by moving forward. Perception \& Psychophysics, 17, 445-449.

Weber, K. D., Fletcher, W. A., Gordon, C. R., Melvill Jones, G., \& Block, E. W. (1998). Motor learning in the "podokinetic" system and its role in spatial orientation during locomotion. Experimental Brain Research, 120, 377-385.

Wertheim, A. H., Mesland, B. S., \& Bles, W. (2001). Cognitive suppression of tilt sensations during linear horizontal self-motion in the dark. Perception, 30, 733-741.

Wiener, S. I., Berthoz, A., \& Zugaro, M. B. (2002). Multisensory processing in the elaboration of place and head direction responses by limbic system neurons. Cognitive Brain Research, 14, 75-90.

Withagen, R., \& Michaels, C. F. (2002). The calibration of walking transfers to crawling: Are action systems calibrated? Ecological Psychology, 14, 223-234.

Wolpert, D. M., \& Flanagan, J. R. (2001). Motor prediction. Current Biology, 11, R729-R732.

Wolpert, D. M., Ghahramani, Z., \& Jordan, M. I. (1995, September 29). An internal model for sensorimotor integration. Science, 269, 1880-1882.

Received November 5, 2003

Revision received August 16, 2004

Accepted September 21, 2004 